Proc. Indian Acad. Sci. (Earth Planet. Sci.), Vol. 95, No. 3, November 1986, pp. 447-484.

(C) Printed in India.

\title{
An overview of climate models
}

\author{
M SANKAR-RAO \\ Department of Aerospace Engineering, and The Centre for Atmospheric Sciences, \\ Indian Institute of Science, Bangalore 560012 , India \\ MS received 11 December 1985; revised 21 April 1986
}

\begin{abstract}
Climate models and results, especially for paleo-climatic scale are reviewed. It is concluded that the climatic system is more stable than it was thought to be when Budyko-Sellers type models first came into existence. Even a $2 \%$ decrease in solar radiation may not result in White Earth due to negative feed-backs. $A$ decrease in $\mathrm{CO}_{2}$ to about $200 \mathrm{ppm}$ can result in White Earth. A doubling of $\mathrm{CO}_{2}$ increases the surface temperature by an order of $1^{\circ} \mathrm{C}$. The total climatic system which is nonlinear can exhibit very long period internal oscillations, even of the order of $1,00,000$ years though none of the time constants involved are in that range individually.
\end{abstract}

Keywords. Climate model; paleo-climatic scale; white earth.

\section{Introduction}

Climate on earth can be defined as the statistical average state of the total earth atmospheric system. The parameter (or parameters) describing such a state must be variable. A conservative quantity in a closed system cannot obviously be such a parameter. The length of time to be considered for averaging depends on the time averages that exist and are observable in reality. Two time scales, one seasonal and the other being a very long paleoclimatic one (about a few centuries) are of great concern to us, because on these time scales the variations are quite significant according to observations. Seasonal climate fluctuations are quite common and well known for years without rains and without summers. On the paleoclimatic scale, there is irrefutable evidence that this earth has experienced an extensive glaciation in the distant past. The climate during such ice ages was crucial to evolution of life on earth. An attempt is made here to survey the existing theories of climate, especially on the paleoclimatic scale. The literature on this subject is so vast that it is almost impossible to review or refer to all the works. Therefore, attention will be focussed on a few quantitative theories typifying the approach.

First, we shall refer to some observations very briefly and then describe the theoretical approach. Later, we discuss various advanced theories and finally, try to indicate the possible future course. The notations are mostly given in appendix $\mathbf{A}$.

\section{Climatic observations}

The seasonal global climate maps of temperature, winds, pressure and humidity at different levels of the atmosphere are too well known for describing this cycle with cold surface highs and warm surface lows over the continents during winter and 
summer seasons respectively. These observations are based on modern upper air and surface observations directly recorded.

Regarding ancient climates, a variety of observational methods such as geological, paleobiological and physical methods are followed. All these methods examine ancient material to study the climate existing then. Briefly the idea behind each method can be stated as follows:

Geomorphological features such as striated surfaces over wide areas of low relief are generally taken as evidences of extensive glaciation in the past. Existence of different kinds of biota in the ancient samples is taken as the evidence of suitable environment for these types of life forms in the period concerned. The oxygen isotope ratio $\left(\mathrm{O}_{18} / \mathrm{O}_{16}\right)$ or the ratios of the isotopes of other suitable elements, in the samples is taken as an indication of temperature because according to Urey's (1947) theoretical analysis carbonates precipitated from a single aqueous solution at different temperatures will have different $O_{1 \times}$ contents (Emilliani 1955).

Figure 1 gives the ice age history of the earth as reconstructed by following all the methods described above. One can see that ice ages are rare phenomena. However, they do not seem to be periodic and hence not easily predicted in advance. Therefore it is necessary to understand the possible causes of such catastrophic climatic events in order to be able to predict them and to know the future of the planet at least till the last stages of the sun.

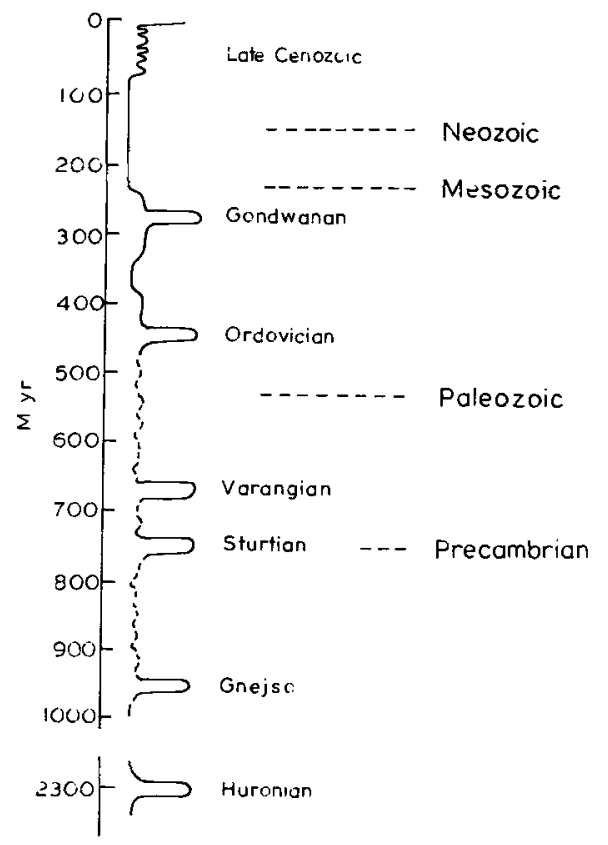

Figure 1. Ice ages through geological time (in millions of years before present) (from Gibbon J 1978). 


\section{The physical basis of climate change}

Figure 2 gives a deceptively simple picture of the earth atmospheric system. One can easily see that the climate is a state of the earth atmospheric system as a result of complex interaction of the atmosphere with its lower boundary and the incoming solar radiation. It is theorized that climatic perturbations can occur either due to external changes such as changes in solar radiation or due to internal changes in albedo, seasurface temperature, cloudcover, atmospheric composition etc. To solve such questions while complex feedback mechanisms are operating, one must take recourse to mathematical modelling. The atmosphere-earth system is replete with many feedback loops. A positive feedback is destabilizing while a negative one is stabilizing. Ice albedo feedback is an example of positive feedback. The greater the ice cover the greater the planetary albedo, and lesser the energy input, lesser the temperature and hence greater the ice cover. Infrared cooling is an example of negative feedback. The greater the temperature, the greater the infrared radiation into space and the cooling, and hence, lesser the temperature. With many such competing and conflicting mechanisms it is obvious that mathematical modelling is

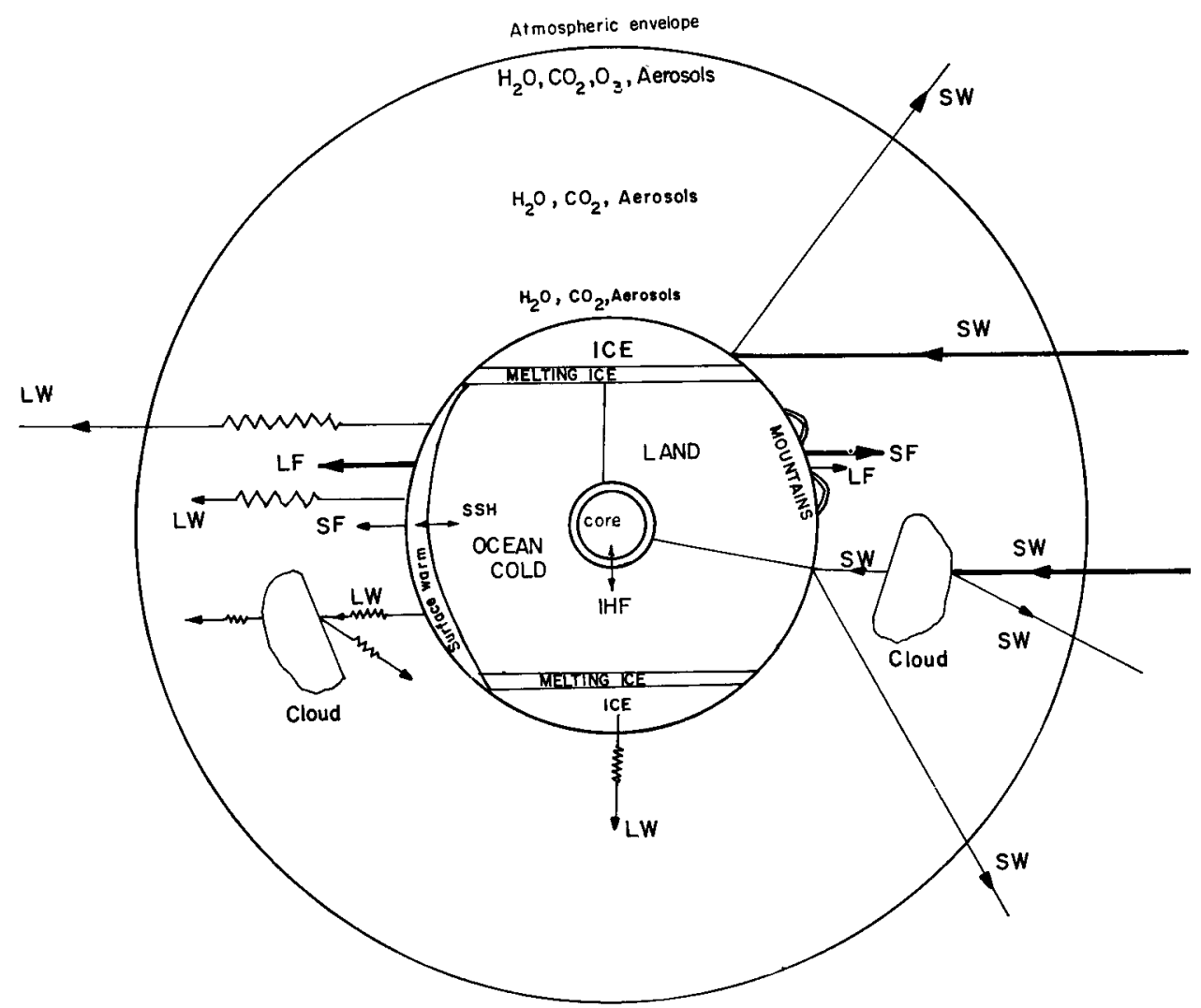

Figure 2. Earth atmosphere radiative system (LW: Long Wave; SW: Short wave radiations; LF: Latent heat flux; SF: Sensible heat flux; SSF: Subsurface heat flux; IHF: Interior heat flux-thick and thin lines represent high and low values respectively. 
the only rational method for the study of such systems. A number of such models have already been proposed and are being tested. We shall first deal with paleoclimatic models. These models can be categorised as (1) zero and onedimensional energy balance models, (2) vertically or zonally averaged twodimensional dynamical models, and (3) three-dimensional dynamical models. We shall now discuss these types of models in that order.

\subsection{The energy balance models}

The basis of all these models is the time-averaged thermodynamic energy equation for the earth (ocean and land) or the atmosphere or for the earth-atmosphere system as a whole. It is written as

$$
C_{m}\left(\frac{\partial}{\partial t} \bar{\rho}^{\prime} \bar{\theta}^{t}+\overline{\nabla \rho \nu \theta^{\prime}}+\frac{\partial}{\partial z} \overline{\rho w \theta^{\prime}}\right) \equiv-\frac{\partial}{\partial z} \bar{F}_{\mathrm{HV}}^{t}-\nabla \bar{F}_{\mathrm{HH}}^{t}
$$

This is the basic equation for three-dimensional models.

The zonal average form of (1) gives one type of two-dimensional model equation as

$$
C_{m} \frac{\partial}{\partial t} \bar{\rho}^{t x} \bar{\theta}^{t x}+\frac{\partial}{\partial y} \overline{\rho \nu \theta}{ }^{t x}+\frac{\partial}{\partial z} \overline{\rho w}^{t x}=-\frac{\partial}{\partial z} \bar{F}_{\mathrm{HV}}^{t x}-\frac{\partial}{\partial z} \bar{F}_{\mathrm{HH} y}^{x x}
$$

If we take the vertical average of (1) we get another kind of two-dimensional model equation as

$$
\begin{aligned}
C_{m} \frac{\partial}{\partial t} \bar{\rho}^{t z} \bar{\theta}^{t z} & +\frac{\partial}{\partial x} \overline{\rho u \theta} \bar{c}^{t z}+\frac{\partial}{\partial y} \overline{\rho \nu \theta^{t z}} \\
& =\bar{F}_{\mathrm{HV}-\mathrm{B}}^{t}-\bar{F}_{\mathrm{HVT}}^{t}-\frac{\partial}{\partial x} \bar{F}_{\mathrm{HH} x}^{t z}-\frac{\partial}{\partial y} \bar{F}_{\mathrm{HH} y}^{t z} .
\end{aligned}
$$

Here we have used the boundary condition that $W=0$ at $Z=-B$ and $Z=Z_{T}$.

If we take the average of ( $2 a)$ over $y$, under the following boundary conditions of

$$
F_{\mathrm{HH} y}=V=0 \text {, at } y \pm Y \text {. }
$$

we get one kind of one-dimensional model equation

$$
C_{m}\left(\frac{\partial}{\partial t} \bar{\rho}^{t x y} \bar{\theta}^{t x y}+\frac{\partial}{\partial z} \overline{\rho w \theta}^{x y}\right)=-\frac{\partial}{\partial z} \bar{F}_{\mathrm{HV}}^{x y}
$$

Another kind of one-dimensional model equation is obtained if we take the vertical average of $(2 \mathrm{a})$ and write

$$
C_{m} \frac{\partial}{\partial t} \bar{\rho}^{t x z} \bar{\theta}^{t x z}+\frac{\partial}{\partial y} \overline{\rho \nu}^{t x z}=\bar{F}_{\mathrm{HV}-\mathrm{B}}^{x}-\bar{F}_{\mathrm{HVT}}^{t x}-\frac{\partial}{\partial y} \bar{F}_{\mathrm{HHy}}^{t x z}
$$


We can write (4) separately for the ocean as

$$
\begin{gathered}
C_{m_{1}} \frac{\partial}{\partial t} \bar{\rho}_{1}^{t x z} \bar{\theta}_{l}^{t x z}+C_{m_{1}} \frac{\partial}{\partial y}{\overline{\rho_{l} \nu_{l} \theta_{l}}}^{t x z} \\
=\bar{F}_{\mathrm{HV}-\mathrm{B}}^{x x}-\bar{F}_{\mathrm{HVO}}^{\prime x}-\frac{\partial}{\partial y} \bar{F}_{\mathrm{HH} y}^{t x z} .
\end{gathered}
$$

For the atmosphere we can write the counterpart of (5) as

$$
\begin{gathered}
C_{m_{2}} \frac{\partial}{\partial t} \bar{\rho}_{2}^{\prime x z} \bar{\theta}_{2}^{x z}+C_{m_{2}} \frac{\partial}{\partial y} \overline{\rho_{2} v_{2} \theta_{2}} d x z \\
=\bar{F}_{\mathrm{H} V \mathrm{O}}^{x}-\bar{F}_{\mathrm{H} \vee \mathrm{T}}^{\prime x}-\frac{\partial}{\partial y} \bar{F}_{\mathrm{HH} y}^{t x z} .
\end{gathered}
$$

Equations (5) and (6) describe many one-dimensional energy balance models. Now, if we take the average over $x, y$ and $z$ of (5) we get zero-dimensional model equations. We can write them as follows:

For the lower surface

$$
\frac{\partial}{\partial t}{\overline{C_{m_{1}} \rho_{1} \theta_{1}}}^{x y z}=\bar{F}_{\mathrm{HV}-\mathrm{B}}^{t x y}-\bar{F}_{\mathrm{HVO}}^{\prime x y}
$$

for the atmosphere

$$
\frac{\partial}{\partial t}{\overline{C_{m_{2}} \rho_{2} \theta_{2}}}^{x y z z}=\bar{F}_{\mathrm{HVO}}^{\prime x y}-\bar{F}_{\mathrm{HVT}}^{x y}
$$

and for the earth atmospheric system as a whole

$$
\frac{\partial}{\partial t}{\overline{C_{m} \rho \theta}}^{x y z}=\bar{F}_{\mathrm{HV}-\mathrm{B}}^{x y}-\bar{F}_{\mathrm{HVT}}^{x y} .
$$

Equations (7), (8) and (9) are the appropriate ones for zero-dimensional models.

In these equations the energy flux is the result of complex physical processes. For instance, the surface energy flux parameter $F_{\mathrm{HV}}$ is composed of five different components of significance. We can thus write

$$
F_{\mathrm{HVO}}=\Sigma F_{\mathrm{HVO}}^{t}, i=1 \text { to } 5 \text {, }
$$

where

$$
\begin{aligned}
& F_{\mathrm{HVO}}^{\mathrm{l}}=F_{\mathrm{SR}}=\text { shortwave radiative net flux; } \\
& F_{\text {HVO }}^{2}=F_{\mathrm{LW}}=\text { longwave radiative net flux; } \\
& F_{\text {HVO }}^{3}=F_{\mathrm{SH}}=\text { sensible heat flux due to small scale turbulence. This is } \\
& \text { zero at the top of the atmosphere, } F_{\mathrm{HVT}}^{3}=0 \text {; } \\
& F_{\mathrm{HVO}}^{4}=F_{\mathrm{LH}}=\text { turbulent latent heat flux due to phase changes of }
\end{aligned}
$$




$$
F_{\mathrm{HVO}}^{5}=F_{\mathrm{SSH}}=\text { sub-surface heat flux. }
$$

Each of these components depends on highly complex physical processes. $F_{\mathrm{SR}}$ and $F_{\mathrm{LW}}$ depend on absorption and scattering properties of the earth and the atmosphere which in turn depend on the composition. Similarly $F_{\mathrm{SH}}, F_{\mathrm{LH}}$ and $F_{\mathrm{SSH}}$ depend on dynamical conditions such as wind velocity, wind shear and static stability. Though we have some fundamental knowledge of these properties, it is by no means adequate yet. Our theories are still developing and as such the fluxes we calculate from the existing theories are most probably approximate. It is outside our scope here to discuss the computational details of these flux calculations.

We have given the broad mathematical framework of these energy balance models. In all these models, or in any model for that matter we have the following components in general:

(a) Predictands, or parameters that are being predicted such as temperature, ice line etc. For these parameters one has to explicitly prescribe from observations and physical reasoning, the initial and boundary conditions.

(b) Internal structural parameters such as the large scale conductivity, emissivities etc which are generally empirically parametrized. These can be variable or fixed, but have some uncertainty about their value because of lack of knowledge.

(c) External parameters such as solar constant, angular velocity of the earth etc, which are determined by nature only, but which are also variable.

(d) Parameters that are invariable such as specific heats, Steffan-Boltzman constant etc.

(e) Finally, unknown stochastic parameters.

The types of questions asked about any system concern the sensitivity and stability of the system defined by the model equations. For example:

(i) For fixed structural and external parameters, say corresponding to the present observed state, what is the nature and sensitivity of the model prediction over a period of time, for infinitesimally small perturbations in the initial state which is treated as the initial control state? This question involves a number of questions regarding prediction, stability and predictability raised and examined by Lorenz (1963).

If, depending on the initial magnitude of the 'small' perturbation, the system remains proportionally close to the control system evolution for all times, the system is considered stable. If it differs from the control climate state by greater and greater 'distance in phase space' as time progresses, it is termed as unstable and hence not easily predictable. In such a case or even in the case of bounded 'distance' the predictability has a limiting time which is the time at which the 'distance' between control state and the perturbed state is equal to the natural distance between any two random states of the control evolution. The questions asked are generally: (a) What is the stability property of the system? (b) What is the predictability of the system? There are many sub-questions like the predictability of different parameters, different scales etc here. But we do not go into such details which are outside our scope.

(ii) For fixed structural and external parameters, what is the nature of evolution of the system for large, but physically meaningful, perturbations of the initial 
conditions? There is no point in introducing perturbations so large that under those conditions even model equations do not hold. For instance if we start with very cold subzero temperatures over the earth, evidently it will be initially ice-covered (perhaps with solidified air), and will be ice-covered for ever. In this type of investigation it is of great interest to see what final states are reached by the system for different initial conditions and whether they are stable for small perturbations? If the system always comes to a single steady statistical state, it is termed as transitive system. If it has no single final steady state it is called an intransitive system. Depending critically on initial conditions if the system has more than one steady state solution, it is termed almost intransitive. Some simple nonlinear systems have this property of multiple equilibria. This classification is given by Lorenz. Nonlinear systems can be almost intransitive and there can be sudden changes from one weather or climate regime to another weather or climate regime, critically depending on the initial conditions. Our interest again will be the free final states within the physically meaningful range.

(iii) For the same external parameters, how does the nature of the system with regard to stability, predictability and final states, change with differing structural parameters? It must be expected that the nature of the system depends very much on the structural parameters. Sometimes some of the physically possible solutions disappear from the physically meaningful range if the structural parameters are changed.

(iv) For the same structural parameters how does the nature of the system change with changing external parameters? Here again perturbations in external parameters should be within physically meaningful limits of interest. There is no interest left if the sun is shut off or made a red giant.

(v) For the same external and structural parameters, how does the nature of the system change for various amplitudes of stochastic forcings? Here the stochastic forcings again must be within physically plausible range. They must be determined from observations.

With this digression into the general structure of mathematical models and the questions of interest we shall now turn our attention to the energy balance models.

\subsection{Zero-dimensional energy balance models}

(i) Schneider and Mass model: To determine whether volcanic dust and sun spot associated variation of incoming solar radiation can explain global temperature variations since $1600 \mathrm{AD}$, Schneider and Mass (1975) applied the earth atmospheric system model given by

$$
\frac{\partial}{\partial t}{\overline{\rho C_{m}}}^{\prime x y z}=-\bar{F}_{\mathrm{HVT}}^{x y}=S_{R}-I_{R}
$$

which corresponds to (9). Here $S_{R}$ is the solar radiation flux into system, and $I_{R}$ is the long wave radiation flux out of the system. The solar radiative flux was further divided into its parts as follows:

$$
S_{R}=\frac{1}{4}\left(\Delta S_{S}+\Delta S_{D}+S_{0}\right)(1-\alpha)
$$


where $\Delta S_{S}=$ variation of $S_{R}$ due to sunspot activity, $\Delta S_{D}=$ variaion of $S_{R}$ due to volcanic dust, $S_{0}=$ normal solar constant, and $\alpha=$ Albedo of the planet. $\Delta S_{D}$ was scaled such that there was $0.5 \%$ decrease in $S_{R}$ during 1963 when Mt. Agung erupted in Bali. For $\Delta S_{S}$ an empirical formula was used:

$$
S_{S}=0.011 \sqrt{N}-0.0006 N \text {, }
$$

where $N$ is the sunspot number.

This model could predict the temperature time series since $1600 \mathrm{AD}$ for the northern hemisphere reasonably well.

(ii) Bryson and Dittberner model-To test the effect of anthropogenic dust and increase in $\mathrm{CO}_{2}$, these authors proposed another zero-dimensional model. The model is for global surface temperature of the earth. But to calculate the energy reaching the ground, a three-layer atmosphere model having, (a) lower troposphere with dust, (b) an upper troposphere which is dust-free but which has clouds near the base and (c) a stratosphere which has variable amount of volcanic dust, was considered.

The transmissivities of these three layers are assumed to be $a, c(1-N)$ and $b$ respectively, where $N$ is equivalent opaque cloud amount. The fractional forward scattering diffuse operators for these layers were taken to be $f_{a}, f_{b}$ and $f_{c}$ respectively. The albedo of the surface was taken as $\alpha_{0}$. Thus following a cascading computation Bryson and Dittberner (1976) expressed direct and diffuse net shortwave radiation reaching the ground as follows:

$$
S_{R}=S_{0}\left(1-\alpha_{0}\right)(1-N) \cdot\left\{a+f_{a}(1-a)\right\}\left\{b+f_{b}(1-b)\right\}\left\{c+f_{c}(1-c)\right\} .
$$

To calculate the net longwave radiation the atmospheric back radiation was assumed to be proportional to the outgoing radiation from the surface. Thus $R_{g}$ is ground radiation $=\varepsilon_{e} \sigma T_{0}^{4}$ and $R_{a}$ is atmospheric radiation $=\varepsilon_{e} \sigma T_{0}^{4}\left(1-\varepsilon_{a}\right)$, where $\varepsilon_{e}=$ surface emissivity, $T_{0}=$ screen temperature, and $\varepsilon_{a}=$ atmospheric emissivity.

The atmospheric emissivity $\varepsilon_{a}$ was taken to be due to that of water vapour $\left(\varepsilon_{w^{\prime}}\right)$, and of $\mathrm{CO}_{2}$ and $\mathrm{O}_{3}\left(\varepsilon_{c}\right)$, a correction emissivity $\left(\varepsilon_{1}\right)$ and emissivity due to all other constituents such as dust etc $\left(\varepsilon_{0}\right)$. Thus

$$
\varepsilon_{a}=\varepsilon_{w^{\prime}}+\varepsilon_{c}+\varepsilon_{0}-\varepsilon_{v},
$$

where $\varepsilon_{w}=0.60 ; \varepsilon_{c}=0.0235 \log \left(\mathrm{CO}_{2}\right)+0.0537 \mathrm{CO}_{2}$ in ppm; $\varepsilon_{\mathrm{v}}=0.12$; and $\varepsilon_{0}=0.06$.

Taking the green house effect of clouds, the authors finally arrive at an expression for the net long wave radiation at the surface.

$$
I_{R}=(1-N) \varepsilon_{e} \sigma T_{0}^{4}\left(1-\varepsilon_{w}-\varepsilon_{c}+\varepsilon_{v}-\varepsilon_{0}\right)+N \varepsilon_{a}\left(\sigma T_{0}^{4}-\sigma T_{c}^{4}\right)\left(1-\varepsilon_{s c}\right),
$$

where $\varepsilon_{s c}=$ net cloud emissivity, and $T_{c}=$ cloud base temperature.

The sum of the sensible and latent heat fluxes $L$ at the surface was given a constant value of 0.6 to $1.0 \mathrm{~W} \mathrm{~m}^{-2}$. Thus finally Bryson and Dittberner write the zero-dimensional model equation corresponding to (9) as

$$
\rho C_{m} \frac{\partial \bar{T}_{0}^{t x y z}}{\partial t}=S_{R}+I_{R}-L
$$


In (11), surface flux is neglected as the model is annual-hemispheric. In this model the transmissivities $a, b, c$ are all of the order of 0.9 . This model was utilized to explain the surface temperature variation from 1890 to 1960 . Some results of the sensitivity analysis of this model are given in table 1.

We notice that this model has no other feedback except negative longwave radiation feedback. Though the sensitivity results show changes in the right direction, in this type of model, the variations in temperatures can be expected to have smooth and continuous dependence on the controlling parameters.

Weare and Snell (1974) treated the atmosphere as a diffuse thin cloud and considered a zero-dimensional model based on (9). Their sensitivity results, summarized comparatively by them and reproduced below in table 2 , show greater stability due to the diffuse thin cloud feedback.

While the sensitivity of Bryson and Dittberner model for $1 \%$ change in solar constant is $2.0 \mathrm{~K}$, it is only $0.7 \mathrm{~K}$ for diffuse thin cloud model of Weare and Snell. This type of differences, which may seem small but which are indeed quite large considering what can happen to the earth atmospheric system by reduction of global temperature by $2 \mathrm{~K}$, highlight the importance of considering various feedbacks physically correctly.

When one considers other feedback mechanisms, interesting changes can occur. For instance if the albedo is made a function of temperature itself (ice albedo effect) the model can be expected to exhibit dramatic changes at some critical initial and boundary conditions as we shall see later. It is quite difficult to include such a

Table 1. Sensitivity results of Bryson \& Dittberner model.

\begin{tabular}{lcc}
\hline Symbol & Value & Sensitivity for 1\% change \\
\hline$S$ & $340.0 \mathrm{~W} \mathrm{~m}^{-2}$ & $\pm 2.00 \mathrm{~K}$ \\
$L$ & $94.1 \mathrm{~W} \mathrm{~m}^{-2}$ & $\pm 1.33 \mathrm{~K}$ \\
$\varepsilon_{0}$ & 0.98 & $\pm 0.73 \mathrm{~K}$ \\
$N$ & 0.40 & $\pm 0.40 \mathrm{~K}$ \\
$\alpha_{0}$ & 0.11 & \pm 0.11 \\
\hline
\end{tabular}

Table 2. Temperature change (in $\mathrm{K}$ ) for a given environmental change by various models (Weare and Snell 1974).

\begin{tabular}{|c|c|c|c|c|c|}
\hline Variation & $\begin{array}{c}\text { Diffuse } \\
\text { cloud model }\end{array}$ & $\begin{array}{c}\text { Budyko } \\
\text { (1969) }\end{array}$ & $\begin{array}{l}\text { Sellers } \\
(1973)\end{array}$ & $\begin{array}{c}\text { Rasool \& } \\
\text { Schneider } \\
\quad(1971)\end{array}$ & $\begin{array}{c}\text { Weatherald } \\
\text { and Manabe } \\
(1975)\end{array}$ \\
\hline Doubling $\mathrm{CO}_{2}$ & $0 \cdot 7$ & $4 \cdot 0$ & $10 \cdot 1$ & 0.8 & $2 \cdot 3$ \\
\hline $\begin{array}{l}\text { Doubling aerosol } \\
\text { optical depth }\end{array}$ & $-1 \cdot 1$ & - & $<-5 \cdot 0$ & 1.8 & - \\
\hline $\begin{array}{l}1 \% \text { decrease in } \\
\text { solar constant }\end{array}$ & -0.7 & $-5 \cdot 0$ & $-5 \cdot 0$ & - & $-1 \cdot 3$ \\
\hline $\begin{array}{l}1 \% \text { increase in } \\
\text { solar constant }\end{array}$ & 0.7 & - & - & 0.9 & $1.3 \mathrm{~K}$ \\
\hline
\end{tabular}


feedback physically correctly in zero-dimensional models because of opposing baroclinicity effects. However, for completeness, we give below the parametrization of such an effect by Weare and Snell within the framework of zero-dimensional models:

$$
\begin{aligned}
& \bar{\phi}_{S}^{x}=65 \cdot 0+0 \cdot 1\left(\bar{T}_{0}^{x y}-288 \cdot 0\right) \\
& \bar{\phi}_{N}^{x}=65 \cdot 0+2 \cdot 0\left(\bar{T}_{0}^{x y}-288 \cdot 0\right) \\
& \bar{\phi}^{x}=0 \cdot 5\left(\bar{\phi}_{S}^{x}+\bar{\phi}_{N}^{x}\right) \\
& \alpha_{S}=\left(1-\sin \bar{\phi}^{x}\right) \alpha_{\text {ice }}+\left(\sin \bar{\phi}^{x}\right) \alpha_{\text {Nonice }},
\end{aligned}
$$

where $\bar{\phi}_{S}^{x}$ is the southern hemispheric ice line, $\bar{\phi}_{N}^{x}$ the northern hemispheric ice line, $\bar{\phi}^{x}$ the mean ice line latitude, $\alpha_{\text {ice }}$ the ice albedo $=0.6, \alpha_{\text {Nonice }}$ the non ice albedo $=0.07, T_{0}$ the surface temperature and $\alpha_{S}$ the surface albedo. The large amount of empiricism of the above models is a common necessity in palaeoclimatic models and so we cannot hold this against these models as long as the parametrizations are done correctly.

The above models are examples of zero-dimensional energy balance models for the earth's surface and are purely radiative in character. Neither dynamics nor convective overturning of the atmosphere is considered. The next natural extension will be to include convective adjustment of the atmosphere also besides the radiative transfer. In effect such radiative convective models are parametrizing $L$, the sensible and latent heat transfer from the surface in a more realistic way by modelling the details of the atmospheric temperature structure. These models in general consider the most important radiatively active gases and aerosols. The radiative transfer calculations are performed by considering the absorption spectrum to various degrees of aproximation. Convective adjustment is applied so that no part of atmospheric column is either dry adiabatically or moist adiabatically unstable. Because these models consider the atmospheric structure in detail in one dimension (vertical) we shall consider them as one-dimensional radiative convective energy balance models.

\subsection{One-dimensional radiative convective energy balance models}

Manabe and Moller model-One of the earliest of detailed radiative models is that of Manabe and Moller (1961). This was a test model to be later attached to a general circulation model for radiative transfer calculations. Moller and Manabe found that without convective adjustment computed tropospheric temperature were much too low because of lack of effective upward transfer of heat. Manabe and Strickler (1964) introduced convective adjustment. Manabe and Wetherald (1967) considered the same model which considers all radiatively important constituents. Their final results could reasonably get the correct lapse rate of $6.5^{\circ} \mathrm{K} / \mathrm{km}$. For details the reader may refer to the original papers.

For the radiative part of these models of this type, the fundamental equation considered is the Schwarzchild's radiative transfer equation for plane parallel atmosphere. This equation can simply be written as 


$$
\begin{aligned}
\cos \theta \frac{\mathrm{d} I_{\nu}}{\mathrm{d} \tau_{R}}= & -I_{\nu}+\mu^{2}\left(1-a_{\nu}\right) B(\nu, T) \\
& +\frac{1}{4 \pi} \int_{0}^{4 \pi} \chi(\cos \gamma) a_{\nu} I_{\nu} \mathrm{d} w .
\end{aligned}
$$

We notice that (13) is an integro-differential equation for a beam of $\nu$ radiation in a plane parallel atmosphere. Thus the history of radiant energy after it enters a medium depends on $\nu, \theta, K_{\nu}, \rho, \sigma, \mu, a_{\nu}, B(\nu, T)$ and $\chi$. Indeed strictly complete radiative transfer computations are extremely difficult, even assuming we know $\chi(\cos \gamma)$, which is itself very difficult to obtain in real cases in nature.

When the single scattering albedo $a_{\nu}=1$, we have pure scattering problem and when $a_{\nu}=0$, we have pure absorption problem. Especially scattering computations as (13) reveals, will be most involved. So it is customary to simplify these computations by empiricism and assuming transmission and scattering coefficients. An example of such a procedure has already been given while discussing the Bryson and Dittberner model.

Scattering can be neglected for infrared calculations of the atmospheric radiative transfer. For short wave calculations, absorption is sometimes neglected while scattering is important. Hence at least for infrared calculations we deal with a simpler equation. Even for short wave region if we assume transmission and scattering coefficients, our equation will be much simpler than (13). Such methods are utilized in almost all atmospheric radiative calculations.

Now one important point remains to be clarified. Equation (13) is for a single $\nu$. How can we solve such an equation for all relevant $\nu$ ? They will be enormous in number. However, because $B(\nu, T)$ varies slowly over short spectral wavenumber intervals (say $100 \mathrm{~cm}^{-1}$ ) the problem is simplified for such short intervals. The next problem is to find the correct absorption coefficient or, absorptivities for this interval. About 20 such intervals are needed to model one absorption band, say $6 \cdot 3 \mu$, of water vapour (Ramanathan and Coakley 1978). Because $K_{\nu}$ varies rapidly with $\nu$ it is difficult to define a single average absorption coefficient for any band. Kondratyev suggested a generalized absorption coefficient usage which can reduce the number of $K_{\nu}$ s to be considered without introducing large errors. The method is based on a shift of emphasis from the frequency to the value of the absorption coefficient for the computation of averages. To get the average absorption coefficients we turn to spectroscopic theory and actual laboratory observations. It is found that $K_{\nu}$ depends on pressure and temperature also, and corrections have to be applied for these effects. To circumvent the detailed computational procedure briefly indicated above, empirical emissivities and absorptivities are generally utilized (Manabe and Moller 1961).

From the above discussion one can see that the total radiative calculations for the atmosphere in all their complexity can never be performed. Even if we perform line-by-line integration the problem of cloud effect with all its uncertainties remains. To consider the finiteness of a single cloud is itself a very challenging problem in a theoretical sense. If one has to consider a number of clouds of different structures and sizes the computational problem tends towards hopeless complexity if attempted purely by scattering theory. Therefore we can easily conclude that at one stage or other we have to take recourse to some level of empiricism in these computations. In such a case we must also know the sensitivity 
of radiative transfer calculations to various empirical factors introduced. Therefore we need to perform highly theoretical computations at least for some simple cases and this is the justification for continuous sophistication in radiative computations.

Emissivity formulation is a very widely used empirical method in atmospheric radiative calculations. The results of Manabe and Moller (1961), using this method are given in figure 3 . The following points can be noted:

(i) $\mathrm{H}_{2} \mathrm{O}$ and $\mathrm{CO}_{2}$ have a cooling effect of the order of $1.5^{\circ} \mathrm{K} /$ day in the infrared.

(ii) They both have a heating effect in the short wave region. $\mathrm{H}_{2} \mathrm{O}$ heating effect is quite considerable, of the order of $0.5^{\circ} \mathrm{K} /$ day, in the lower troposphere where $\mathrm{H}_{2} \mathrm{O}$ is maximum.

(iii) In stropospheric regions $\mathrm{O}_{3}$ is the most important constituent contributing to the heating effect of about $2^{\circ} \mathrm{K}$.

Other radiative convective models suggested are similar to that of Manabe and Moller. In these models clouds are generally climatological and fixed. The distribution of $\mathrm{CO}_{2}$ and $\mathrm{O}_{3}$ are also fixed in many models. For $\mathrm{H}_{2} \mathrm{O}$ it is sometime assumed that the relative humidity is only a function of surface relative humidity and pressure

$$
\mathrm{RH}=\mathrm{RH}_{*} f(p),
$$

convective adjustment is invariably applied.

One of the most important results to emerge from these models is that for the surface temperature of the planet, the hierarchy of importance in constituents is, clouds, $\mathrm{H}_{2} \mathrm{O}, \mathrm{CO}_{2}$, and $\mathrm{O}_{3}$ in that order. This hierarchy varies for other temperatures at other altitudes. Climatic experiments have been performed by many workers with such one-dimensional models of radiative convective type. (Manabe and Strickler 1964; Manabe and Wetherald 1967; Rasool and Schneider 1971; Weare and Snell 1974; Schneider 1975; Ramanathan 1976; Wang et al 1976; Ramanathan and Coakley 1978). The most consistent results of such experimentation are given in table 3 .

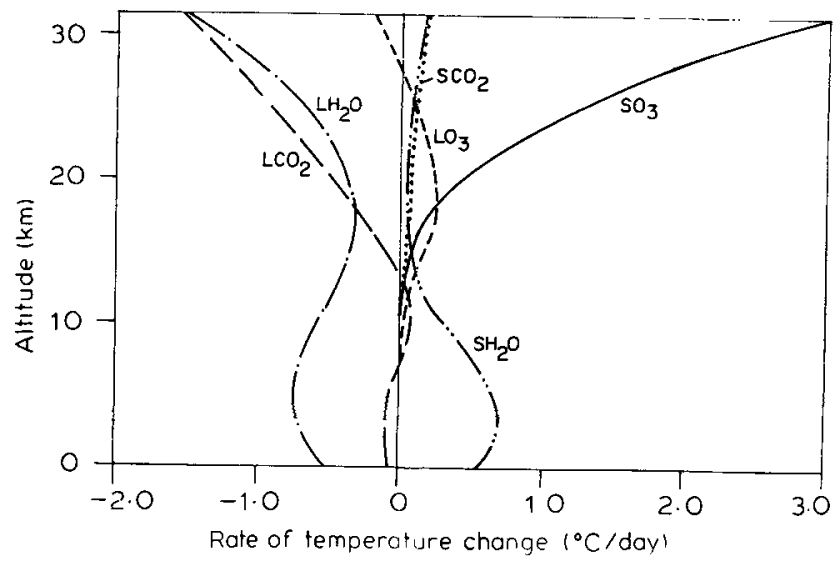

Figure 3. Vertical distribution of the various heat balance components corresponding to the radiative quasi equilibrium ( $\mathrm{L}=$ long wave, $\mathrm{S}=$ short wave) (from Manabe and Moller 1961). 
Table 3. Sensitivity results of some radiative convective models

\begin{tabular}{|c|c|c|c|}
\hline Parameter & $\begin{array}{c}\text { Change } \\
(\%)\end{array}$ & $\begin{array}{c}\text { Surface } \\
\text { effect }(K)\end{array}$ & $\begin{array}{l}\text { Stratospheric } \\
\text { effect (K) }\end{array}$ \\
\hline $\begin{array}{ll}\mathrm{CO}_{2} & \\
\mathrm{O}_{3} & \\
\text { Clouds: } & \text { Low } \\
& \text { Medium } \\
& \text { High }\end{array}$ & $\begin{array}{l}\text { Doubling } \\
\quad-50 \\
+1 \\
+1 \\
+1\end{array}$ & $\begin{array}{l}+2.0 \\
-0.5 \\
-0.82 \\
-0.39 \\
-0.17\end{array}$ & $\begin{array}{l}-8 \cdot 0 \\
-5.0\end{array}$ \\
\hline Solar constant & \pm 1 & \pm 1.3 to \pm 1.6 & \\
\hline
\end{tabular}

Though the radiative convective models deal with detailed structure of the atmosphere in the vertical direction, it is difficult to consider feedbacks of great importance which have something to do with planetary albedo. This is because albedo arising mainly due to ice or snow cover and clouds is a function of latitude and longitude. Even if one tries to relate surface albedo and cloud cover to the surface temperature in these models, it will be physically not satisfactory. Suppose one relates the surface albedo to surface temperature such that cooler temperatures are associated with greater ice cover and hence greater albedo, it will not be correct without considering the baroclinicity and dynamical effects. It is because the large scale dynamical feedback mechanism is always a negative feedback as far as temperature gradients are concerned and so opposes the advance of ice cover. Greater the ice cover, greater the baroclinicity and hence the heat transport to the poles and consequently lesser the ice cover. Here is a powerful negative feedback mechanism which is due to the large scale dynamics.

In any case the vertical structure of the atmosphere is controlled by convection to a great extent. Hence the surface temperature fixes the temperature of the system. This surface temperature depends on the surface energy balance, which in turn depends on radiative transfer. However, it is felt that these energy balance parameters at the surface can be parametrized in terms of surface temperatures (without actually computing radiative fluxes in detail) with sufficient accuracy. Such a possibility allows one to construct one-dimensional models in the $y$ direction (latitudinal direction) and consider the dynamical effects and the latitudinal variation of the albedo. Thus we are led to the latitudinal one-dimensional energy balance models. We shall now deal with such models.

\subsection{Latitudinal one-dimensional energy balance models}

Budyko-Sellers type models-Equations (4), (5) and (6) are the basic equations for these models. The models differ in parametrization of various terms in this equation. Budyko (1969) and Sellers (1969) proposed two simple models based on (4) to (6) and concluded that the climate of earth is not as stable as it appears. Both the models predicted a deepfreeze for a comparatively small decrease of solar radiation $(1-1.5 \%)$ which can easily result due to anthropogenic disturbances. This result was a major reason for a great spurt of interest in climate theory in recent 
years. We shall now deal with Budyko's model with the following assumptions introduced in (5) and (6):

$$
\begin{aligned}
& \bar{F}_{-B}^{t x}=0 \\
& \bar{F}_{\mathrm{HH} y}^{\mathrm{txz}}=0, \\
& \overline{\rho v_{1} \theta_{1}} t x z=0, \text { and } \\
& C_{m_{2}} \frac{\partial}{\partial y} \overline{\rho_{2} v_{2} \theta_{2}} t x z=\bar{F}_{\mathrm{HVO}}^{t x}-\bar{F}_{\mathrm{HVT}}^{x x} .
\end{aligned}
$$

Budyko started with a simplified equation (in the light of the above assumptions) corresponding to (5),

$$
\begin{gathered}
C_{m_{1}} \frac{\partial}{\partial t} \bar{\rho}_{1}^{t x z} \bar{\theta}_{1}^{t x z}+C_{m_{2}} \frac{\partial}{\partial y} \overline{\rho_{2} v_{2} \theta_{2}}{ }^{x x z} \\
=-\bar{F}_{\mathrm{HVT}}^{t x}=\bar{F}_{\mathrm{HVT}}^{t x_{1}}=\bar{F}_{\mathrm{HVT}}^{t x_{2}} .
\end{gathered}
$$

Here $\bar{F}_{\text {HVT }}^{z x_{1}}$ and $\bar{F}_{\text {HVT }}^{c x_{2}}$ are fluxes at the top due to solar and infrared radiation respectively. Based on empirical computations over a large number of meteorological stations, Budyko assumed

$$
\begin{aligned}
& \bar{F}_{\mathrm{HVT}}^{t x_{1}}=-S(1-\alpha), \\
& \bar{F}_{\mathrm{HVT}}^{t x_{2}}=a+B \bar{\theta}_{1}^{t x z}-\left(a_{1}+B_{1} \bar{\theta}_{1}^{t x z}\right) n, \\
& C_{m_{2}} \frac{\partial}{\partial y} \overline{\rho_{2} v_{2} \theta_{2}} t x z=\beta\left(\bar{\theta}_{1}^{t x z}-\bar{\theta}^{t x y z}\right),
\end{aligned}
$$

Here $\alpha$ is the Albedo of the planet; $\beta$ an empirical constant $=0.235 \mathrm{~K} \mathrm{cal} \mathrm{cm}^{-2}$ month ${ }^{-1} \mathrm{~K}^{-1} ; S$ is the solar constant $=1.92 \mathrm{cal} \mathrm{cm}^{-2} \mathrm{~min}^{-1} ; a, B, a_{1}, B_{1}$ are empirical constants equal to $14 \cdot 0,-0 \cdot 14,-3 \cdot 0,-0 \cdot 1$ respectively and $n=$ cloud cover. With these assumptions Budyko writes (14) as

$$
\begin{aligned}
& C_{m_{1}} \frac{\partial}{\partial t} \bar{\rho}_{1}^{t x z} \bar{\theta}_{1}^{t x z}+\beta\left(\bar{\theta}_{1}^{t x z}-\bar{\theta}_{1}^{t x y z}\right) \\
& \quad=S(1-\alpha)-a-\beta \bar{\theta}_{1}^{t x z}+\left(a+B_{1} \bar{\theta}_{1}^{t x z}\right) n
\end{aligned}
$$

with temperature-dependent albedo

$$
\begin{gathered}
\alpha=0.62 \text { if } T<-10^{\circ} \mathrm{C} \text { is covered } \\
0.50 \text { if } T=-10^{\circ} \mathrm{C} \text { ice line } \\
0.32 \text { if } T>-10^{\circ} \mathrm{C} \text { ice free }
\end{gathered}
$$

and the planetary temperature

$$
\bar{\theta}_{1}^{x x y z}=\frac{\bar{S}^{y}\left(1-\bar{\alpha}^{y}\right)-a_{1}+a_{1} n}{B-B_{1} n} .
$$

Because of albedo ( $\alpha$ ) dependence given by (16) on temperature, (15) becomes a nonlinear climate equation. 
Sellers (1969) proposed a similar model differing from Budyko's model, especially in the parametrization of large scale eddy fluxes. Sellers takes the Austauch approach and writes

$$
-\frac{\partial}{\partial y} \overline{v_{2} \theta_{2}} t x z=\frac{\partial}{\partial y} K \frac{\partial}{\partial y} \bar{\theta}_{2}^{t x z}
$$

where $K$ is a macro turbulence coefficient of the order of $10^{6} \mathrm{~m}^{2} \mathrm{sec}^{-1}$, taken as a constant.

The albedo in Sellers model was also a function of surface temperature and is given by:

$$
\begin{gathered}
\alpha=b-0.009 \bar{\theta}_{1}^{t x z} ; \text { if } \bar{\theta}_{1}^{t x z}<283 \cdot 16 \\
b-2.458 ; \quad \text { if } \bar{\theta}_{1}^{t x z}>283 \cdot 16,
\end{gathered}
$$

where $b$ is a function of latitude only. The infrared radiative term in Seller model is nonlinear and is given by

$$
\bar{F}_{\mathrm{HVT}}^{t x z}=\sigma\left(\bar{\theta}_{1}^{t x z}\right)^{4}\left(1-m \tanh \left\{19\left(\bar{\theta}_{1}^{t x z}\right)^{6} 10^{-16}\right\}\right),
$$

where $\sigma$ is the Stefan-Boltzman constant, and $m$ is the Atmospheric attenuation coefficient $=0 \cdot 50$. One can see that both these models parametrize entire dynamics in simple ways. However, especially in the parametrization of large scale eddy flux and the long wave flux, these models differ considerably. Yet both these models gave similar results. Budyko's results are shown in figure 4. We can see that the ice line latitude decreases non-linearly with decreasing solar radiation. Once the ice line crosses a critical latitude, the ice spreads by self-development until it covers the whole earth. Similar result was obtained by Sellers also, who improved his model

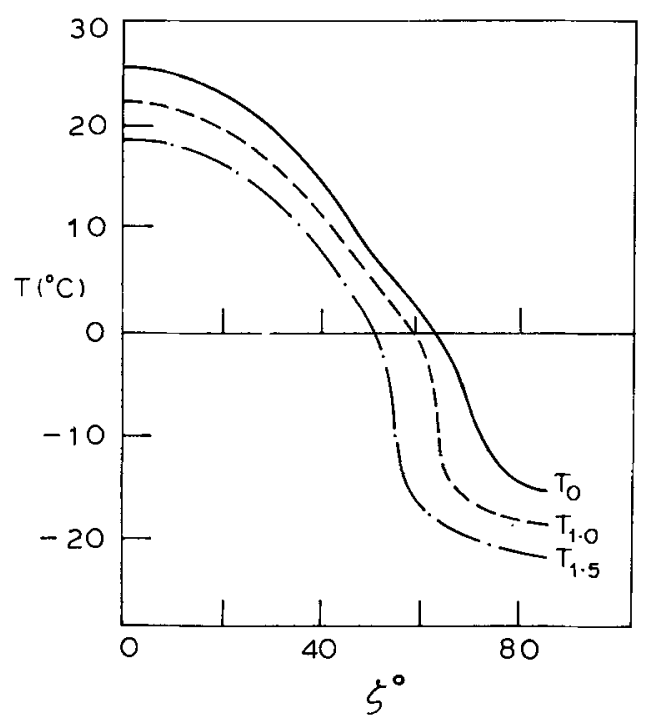

Figure 4. The dependence of temperature $(T)$ distribution with latitude $(\xi)$ on radiation amount. The suffixes for $\mathrm{T}$ indicate the percentual decrease in solar radiation (from Budyko, 1969). 
further e.g. Sellers (1973). Such an astonishing result by sufficiently different models generated a great interest in these "educational toy" models. Therefore Budyko-Sellers type models were further investigated by a number of workers. Feagre (1972), Schneider and Gal-Chen (1973), Dwyer and Petersen (1973), Chylek and Coakley (1975), Held and Suarez (1974), Schneider and Dickinson (1974), North (1975a, b), Cess (1976), Su and Hsieh (1976), Ghil (1976), Cahalan and North (1979), North and Coakley (1979) and others examined the steady state behaviour, sensitivity, internal and structural stability of Budyko-Sellers type models and their minor variants. The temperature-dependent albedo formulation of all these models can be given in a generalized form (Ghil 1976) as

$$
\alpha=\left|b(\phi)-C_{1}\left(\bar{\theta}_{1 m}^{x y z}+\left(\bar{\theta}_{1}^{t x z}-C_{2} Z(\phi)-\bar{\theta}_{1 m}^{t x y z}\right)\right)_{-}\right|_{C}
$$

where $b(\phi)$ and $Z(\phi)$ are given functions of the latitude; $C_{1}$ and $C_{2}$ are empirical constants (0.009 and $0.0065 \mathrm{~K}^{-1}$ respectively); $C$ stands for cut-off; $D$ is any dependent variable; $D_{-}^{=\min }(D, 0) ; D_{C}=0.25$ if $D \leq 0.25 ; D_{C}=D$ if $0.25<D<0.85$; $D_{C}=0.85$ if $0.85 \leq D$; and $m$ stands for yearly average.

In the above type of formulation with $\bar{\theta}_{1}^{t x z}-C_{2} Z(\phi)>\bar{\theta}_{1}^{t x y z}$ (when the temperature is greater than an average temperature), $\alpha$ is independent of the surface temperature because snow or ice cannot be expected at these latitudes above a certain critical temperature. Almost all the albedo formulations generally used are generalized by (21) by Ghil (1976). Cloud effect can be further included as was done by Mokhov (1979).

The most important results of further investigations into the nature of Budyko-Sellers models with the albedo formulation of the type (21) can be briefly stated as follows:

(i) Steady state behaviour. These models generally predict, for the present-day solar constant, three steady state solutions for the surface temperature at the equator in the range of $100 \mathrm{~K}$ to $300 \mathrm{~K} \mathrm{e.g.} \mathrm{Ghil} \mathrm{(1976),} \mathrm{Frederiksen} \mathrm{(1976).} \mathrm{One} \mathrm{of}$ these solutions $\bar{\theta}_{1 E}^{\text {txyz }}$ corresponds to deep freeze. The second solution $\bar{\theta}_{1 E}^{\text {txyz }}$ corresponds to glacial and the third solution $\bar{\theta}_{1 E}^{z x y z}$ corresponds to the present climate. The three steady state solutions obtained by a few authors are given below for comparison and to get an idea of the numbers involved.

In a time-dependent model such multiple states mean that the final solution is critically dependent on the initial conditions.

Looking at table 4 , one can see great similarity in results. However, one cannot conclude that there is comfortable quantitative concurrence. Such differences, evidently due to differing parametrizations, highlight the need for greater observational work to parametrize the most important terms more physically accurately.

(ii) Stability behaviour. North $(1975,1979)$ and Su and Hsieh (1976) and Mokhov (1979) studied the stability properties of these models and rigorously established what is known as the slope stability theorem. According to this theorem the solution is stable or unstable if

$$
\frac{\mathrm{d} S}{\mathrm{~d} \bar{X}_{S}}>0-\text { stable; and } \frac{\mathrm{d} S}{\mathrm{~d} X_{S}}<0-\text { unstable }
$$


Table 4. Steady state solutions of Budyko-Sellers type models (global means)

\begin{tabular}{llccc}
\hline & & $\bar{\theta}_{1 E}^{t x z_{1}}$ & $\bar{\theta}_{1 E}^{t x z_{2}}$ & $\dot{\theta}_{1 E}^{c x z_{3}}$ \\
\cline { 3 - 5 } Model & \multicolumn{1}{c}{ Authors } & $\begin{array}{c}\text { Deep freeze } \\
(\mathrm{K})\end{array}$ & $\begin{array}{c}\text { Glacial } \\
(\mathrm{K})\end{array}$ & $\begin{array}{c}\text { Inter glacial } \\
(\mathrm{K})\end{array}$ \\
\hline Sellers & Ghil (1976) & $175 \cdot 4$ & $268 \cdot 6$ & $287 \cdot 7$ \\
Faeger & Schneider and & $175 \cdot 0$ & $287 \cdot 0$ & $302 \cdot 0$ \\
& Gal-Chen (1973) & & & \\
& Frederickson (1976) & & & $289 \cdot 0$ \\
\hline \multirow{2}{*}{ Budyko } & Frederickson (1976) & $222 \cdot 0$ & $269 \cdot 0$ & 2890 \\
\hline
\end{tabular}

where $S$ is the solar constant; and $X_{S}$ the sine of latitude of the ice line.

These results were graphically depicted by Cahalan and North (1979) and reproduced in figure 5 . It is seen that the ice free and deep freeze solutions are very stable. Even the present-day climate $\left(X_{S}=0.85\right)$ is quite stable. Very small ice cap solutions seem to be unstable.

Both Budyko and Sellers' models predict deep freeze for $1.5 \%$ reduction in solar radiation. We notice that both these models do not include cloud feedback as pointed out by Bryson and Snell or ice insulation feedback as pointed out by Newell (1974), Bunker (1976) and Saltzman (1978). One of the interesting models in the same class of Budyko-Sellers models is that of Saltzman (1978) (in that it is also an energy balance, nonlinear one-dimensional latitudinal model) which considers one of the important negative feedback mechanisms, the ice insulation feedback mechanism. We shall now deal with this model briefly.

Saltzman's oscillator model. Saltzman (1978) advanced a simple energy balance model with the ice insulation negative feedback having two predictive parameters (1) the mass mean ocean temperature $\theta_{0}$ and (2) the sine of the latitude of ice edge $\eta$. Saltzman and Moritz (1980) further developed this model to include $\mathrm{CO}_{2}$ feedback effects and more realistic turbulent heat transfer at the interface of the earth and the atmosphere. This model is still being developed.

The simplest physical system of Saltzman can be described if we take $y=$ sine of latitude, as follows:

Ocean from $y=0$ to $y=\eta=$ melting ice edge;

Melting ice from $y=\eta$ to $y=\eta+\Lambda=$ frozen ice edge; and Ice from $y=\eta+\Lambda$ to $1=$ pole.

The thicknesses of ice and the active ocean are taken as constants $I$ and $D$ respectively. Frozen ice acts as an insulator so that the sensible heat-transfer cannot take place across it. Across the melting ice, however, the sensible heat-transfer can take place. With this physical picture, Saltzman writes two equations, one for the $\theta$ and one for $\boldsymbol{\eta}$ as follows

$$
\mathrm{d} \theta / \mathrm{d} t=\frac{1}{C_{W} \rho_{W} D} \int_{i=1-4}^{\eta} \bar{F}_{\mathrm{HV}}^{t x} \mathrm{~d} \xi-\int_{\eta}^{1} \bar{F}_{\mathrm{HV}_{3}}^{t x} \mathrm{~d} \xi-\frac{L_{f} \Lambda}{2} M(\eta)
$$




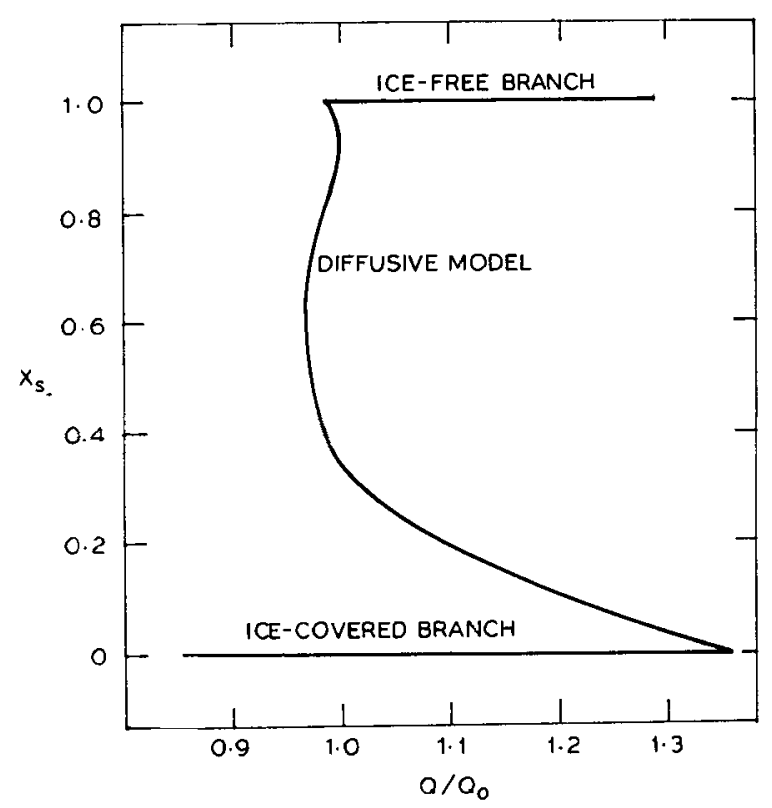

Figure 5. Graph of the sine of the latitude of the ice line $X_{\mathrm{S}}$ as a function of the solar constant $Q$ (in units of the present value $Q_{0}$ ) obtained from a zonal model having diffusive heat transport (from Cahalan and North, 1979).

$$
\mathrm{d} \eta / \mathrm{d} t=\frac{\Lambda}{2 \rho_{\text {ice }} I} M(\eta)
$$

where $C_{W}$ is the specific heat of ocean waters; $\rho_{W}$ is the density of ocean waters; $L_{f}$ is the latent heat of fusion; $\rho_{\text {ice }}$ is the density of ice; and $M(\eta)=$ rate of melting

$$
=\frac{1}{L_{f}} \sum_{i=1}^{5} \bar{F}_{\mathrm{HV},}^{t x} \text { at } y=\eta
$$

After parametrizing for all the heat fluxes Saltzman gets an equation system in the simplest case as follows:

$$
\begin{aligned}
& \mathrm{d} \theta / \mathrm{d} t=-\psi_{1} \eta-\psi_{3} \eta^{2} \theta, \\
& \mathrm{d} \eta / \mathrm{d} t=\phi_{1} \theta-\theta_{3} \eta,
\end{aligned}
$$

where $\psi_{1}, \psi_{3}, \phi_{1}$ and $\phi_{3}$ are positive constants. The system (24) and (25) has two basic components: (i) A damped harmonic oscillation due to ice insulator effect (negative feedback). As $\eta$ increases, $\theta$ decreases due to greater sensible heat loss, and so $\eta$ tends to decrease. (ii) A nonlinear restorative mechanism by $\eta^{2} \theta$ term.

The most important result of Saltzman et al (1980) for a particular variant of his original model that included $\mathrm{CO}_{2}$ feedback is shown in figure 6 . These results are for fixed structural and external parameters and are for arbitrary initial conditions which are small perturbation states from a reference state. $\theta_{0}=278$ and $\eta_{0}=0.85$ approximately. These results can be summarized as follows:

(i) The solution represents a periodic climate, exhibiting a limit cycle behaviour. The major period of oscillation is about 1300 years. The system is intransitive since 

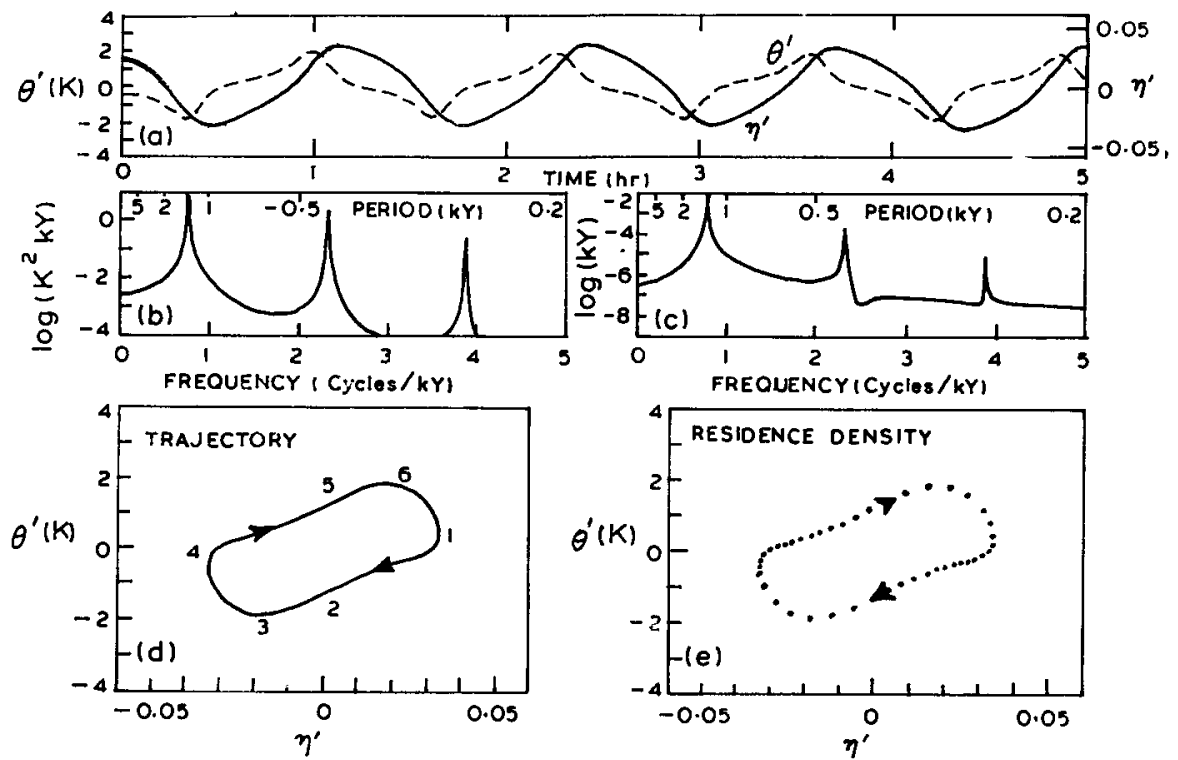

Figure 6. Sample solution for the deterministic system: a) the time evolution of $\eta^{\prime}$ (solid) and $\theta^{\prime}$ (dashed), b) variance spectra for $\theta^{\prime}$, c) variance spectra for $\eta^{\prime}$, d) phase plane trajectory and e) residence density of the solutions (dots are speced at every 30 years) (from Saltzman et al 1981).

it never settles down to a single steady state.

(ii) The system has large residence densities in two states of extreme excursions of $\eta$. These two states can be looked upon as glacial and nonglacial climates. These states correspond to the two states of Budyko-Sellers type models (Cahalan and North).

(iii) $\eta$ lags behind $\theta$ by about a hundred years.

(iv) The amplitudes of $\theta$ and $\eta$ are about $1^{\circ} \mathrm{K}$ and $0.05 \quad\left(=6^{\circ}\right.$ latitude) respectively.

(v) We can say that the ice advances or retreats with a period of 1300 years about a mean position of $58^{\circ}$ latitude. This is about the right period for inter glacial oscillations.

Besides this reference state, about which limit cycle oscillations occur, Saltzman et al found another steady state solution, an unstable saddle point state for large perturbation. This unstable state corresponds to $\eta=0.6469$ (42 latitude) and $\theta=277.67^{\circ} \mathrm{K}$.

Another important finding was that these two states merge at a bifurcation point as the solar radiation is reduced by about $1 \cdot 6 \%$. At this point deep freeze is a possibility. This result agrees with that of Budyko-Sellers model though Saltzman's model is different from Budyko-Sellers type models! Saltzman also obtained a deepfreeze solution for a decrease in $\mathrm{CO}_{2}$ to about $215 \mathrm{ppm}$.

It was found by Saltzman that for the present-day solar constant, stochastic forcings of certain amplitude tend to keep the climate of the model in either of the two extreme excursion states (glacial or nonglacial) of large residence density for comparatively very long time skipping from one state to the other very fast. Hence 
the stochastic forcing of right magnitude makes a limit cycle type intransitive climate model into almost intransitive climate model! Such stochastic forcings can be due to "weather" factors. This result necessitates the correct knowledge of the magnitude of the stochastic forcings in nature.

Here we shall take a detour and dwell a little on what are known as stochastic climate models. Hasselman (1976), Lemke (1977), Reynolds (1978), Ruiz and Lemke (1982) and others are experimenting with this approach. The basic idea here is that for small deviations from an equilibrium state the equation governing a climate variable, say $y_{S}$ (may be SST, ice line etc) is governed by an equation of the type

$$
\mathrm{d} y_{S} / \mathrm{d} t=-V(t) y_{S}(t)+W_{S}(t)
$$

Here $W_{S}(t)$ is the random forcing by weather, $V(t)$ is a structural negative feedback parameter determined mainly by observations. This represents a resistance to change in $y_{S}(t)$. Equation (26) is known as Langevin type of equation. The main problem of these models is to fix correctly the nature of $V(t)$ and $W_{S}(t)$ from observations. These models are being successfully employed to predict SST over a time period of a few months to few years. The signature of $y_{S}(t)$, its amplitude and phase depends critically not only on $V(t)$ but also on $W_{S}(t)$. Thus from the signatures in nature we can reverse compute $V(t)$ and $W_{S}(t)$, having known the behaviour of this model for a variety of combinations of $V(t)$ and $W_{S}(t)$.

Now comparing Saltzman's results with those of Budyko-Sellers type model results, we note the following: (i) Both predict deep freeze for about $-1.5 \%$ perturbation in the solar constant. (ii) Slope stability theorem holds for both the models (Saltzman 1980) (figure 11). (iii) For Budyko-Sellers models the present climate for the present solar constant is a stable one while for Saltzman model it is a nearby climate to a reference climate about which limit cycle oscillation, of period of 1500 years and an amplitude of $6^{\circ}$ latitude for ice line exists. The amplitude of $\theta$ oscillation is only about $1^{\circ} \mathrm{K}$.

The next natural variation of the climate models is to introduce either longitudinal dimension to include land ocean contrast effects also or to introduce the vertical dimension for zonally averaged models to include the atmospheric effects explicitly. In building up the climate models one must be able to keep the model simple enough by correct parametrization or else one will end up with a G.C.M. The real problem of parametrization is more difficult than direct numerical integration. For such parametrizations we must depend on nature and the G.C.Ms. These constraints were kept in mind by the modellers of two-dimensional palaeoclimate models. Now we shall deal with a few typical two-dimensional climate models.

\section{Two-dimensional climate models}

One of the unique two-dimensional models of its kind is due to Sellers (1976). The main equation of this model is $(2 b)$ which can be easily and separately written for ocean, atmosphere and land on which advective fluxes and storage term are neglected. (2b) was applied to a land-ocean atmospheric system by Sellers. The 
main difficulty in applying (2b) is to parametrize the advective part. Sellers utilized the following few of the important assumptions to finally connect all the advective fluxes to the surface temperatures $\theta_{S}$. Denoting atmosphere and oceans by subscripts 1 and 2 respectively Sellers writes for the atmosphere as

$$
\begin{aligned}
& \theta_{1}=\theta_{1 \mathrm{~s}}\left(p / p_{\mathrm{s}}\right)^{0.19} ; \\
& u_{1}=u_{1 \mathrm{~s}}-\frac{g z}{f \theta_{1 \mathrm{~s}}} \frac{\partial \theta_{1 \mathrm{~s}}}{\partial y} ; \text { and } \\
& v_{1}=v_{1 \mathrm{~s}}\left(2 P-P_{\mathrm{s}}-P_{T}\right) /\left(\mathrm{P}_{\mathrm{s}}-P_{T}\right)
\end{aligned}
$$

To compute $u_{0} \mathrm{~s}$ and $v_{0} s$ he considered the Ekman layer equations which also contain the pressure gradients in addition to $u_{0} \mathrm{~s}$ and $v_{0} \mathrm{~s}$. These pressure gradients were expressed by Sellers in terms of surface temperature gradients. For this he utilized a parameter $b$ which is purely empirical. Thus he writes

$$
\frac{\partial p_{\mathrm{s}}}{\partial y}=\frac{b}{a_{2}} \frac{\partial \theta_{0 \mathrm{~s}}}{\partial y}
$$

where $b$ is the Ferrel cell in the middle latitudes. Fixing $b$ is like fixing the geographical extent of the Ferrel Cell. For oceans he takes

$$
\begin{aligned}
& v_{2}=v_{2 \mathrm{~s}} e^{-a z}(1+a z) C ; \\
& \theta_{2}=\theta_{2 W B}+\left(\theta_{2}-\theta_{2 W B}\right) e^{a z} ; \text { and } \\
& v_{2 \mathrm{~s}}= \pm C u_{1 \mathrm{~s}} .
\end{aligned}
$$

Here $\theta_{2 W B}$ is the prescribed subsurface temperature of the ocean.

$$
\begin{aligned}
& \overline{v_{1} \theta_{1}}=\overline{v_{1}} \cdot \overline{\theta_{1}}+\overline{v_{1}^{\prime} \theta_{1}^{\prime}}=\left(\overline{v_{1} \theta_{1}}-K_{1} \frac{\partial \theta_{1}}{\partial y}\right), \\
& \overline{v_{2} \theta_{2}}=\overline{v_{2}} \cdot \overline{\theta_{2}}+\overline{v_{2}^{\prime} \theta_{2}^{\prime}}=\left(\overline{v_{2} \theta_{2}}-K_{2} \frac{\partial \theta_{2}}{\partial y}\right) .
\end{aligned}
$$

Here overbar denotes $-t x z . u_{1}$ is geostrophic in the atmosphere and hence $f_{3}(P)$ was taken consistent with $f_{1}(P) ; u_{1 s}$ and $v_{1 s}$ were determined from Ekman equations for the atmospheric boundary layer with approximations. Note that $v_{1} \cdot \theta_{1}$ and $v_{2} \cdot \theta_{2}$ terms are explicitly taken by Sellers. This is important to include Hadley Cell effects properly in tropics e.g. Lindzen and Farrel 1977, Saltzman and Vernekar (1983).

After utilizing a few more assumptions in parametrizing the solar and infrared radiation fluxes, Sellers could integrate the final equation which is for the surface temperature, with a time step of one month.

Sellers condu'ted some of the standard palaeoclimatological sensitivity experiments with this model. Possible multiple states for the present-day solar constant are indicated by the experiments. These results are reproduced in figure 7 . From this figure one can conclude that the final state reached is a function of initial conditions. 


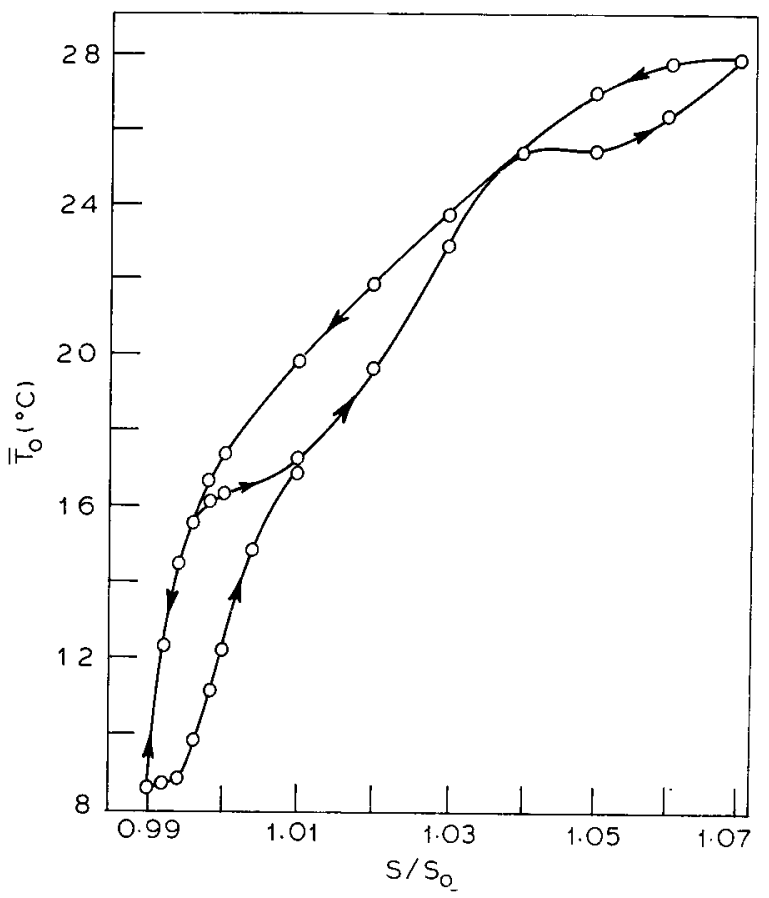

Figure 7. The average global sea level temperature in August as a function of the solar constant $S$, expressed as a function of its present value $S_{0}\left(1.94 \mathrm{ly} \mathrm{min}^{-1}\right)$. The arrows indicate the path followed in increasing or decreasing the solar constant (from Sellers, 1973).

Another typical two-dimensional model is the zonally averaged model. There are a great variety of zonally averaged models to explain the present-day climate e.g. Smagorinsky (1963), Saltzman and Vernekar (1971), Kurihara (1973), Sela and Wiin-Nielsen (1971) and a number of others. For brevity we deal only with the most important aspects of a few models only here.

In all these models parametrization of the large scale turbulent fluxes is the most challenging problem. It is nothing else but a problem in large scale turbulence. If we indicate as usual $u, v, w, \theta, \varepsilon$ and $\phi$ as dependent variables, the eddy flux matrix elements can be written as follows (table 5):

Table 5. Eddy flux parameters.

\begin{tabular}{ccccccc}
\hline & $u$ & $v$ & $w$ & $\theta$ & $\varepsilon$ & $\phi$ \\
\hline$u$ & $\overline{u^{\prime}} 2$ & & & & & \\
$v$ & $\overline{u^{\prime} v^{\prime}}$ & $\overline{v^{\prime 2}}$ & & & & \\
$w$ & $\overline{u^{\prime} w^{\prime}}$ & $\overline{v^{\prime} w^{\prime}}$ & $\overline{w^{\prime}} 2$ & & & \\
$\theta$ & $\overline{u^{\prime} \theta^{\prime}}$ & $\overline{v^{\prime} \theta^{\prime}}$ & $\overline{w^{\prime} \theta^{\prime}}$ & $\overline{\theta^{\prime}}$ & & \\
$\varepsilon$ & $\overline{u^{\prime} \varepsilon^{\prime}}$ & $\overline{v^{\prime} \varepsilon^{\prime}}$ & $\overline{w^{\prime} \varepsilon^{\prime}}$ & - & $\overline{\varepsilon^{\prime}}$ & \\
$\phi$ & $\overline{u^{\prime} \phi^{\prime}}$ & $\overline{v^{\prime} \phi^{\prime}}$ & $\overline{w^{\prime} \phi^{\prime}}$ & - & - & $\bar{\phi}^{\prime 2}$ \\
\hline
\end{tabular}


Here the primed quantities denote eddy fluxes. The most important of these fluxes, for parametrization problems are $\overline{v^{\prime}}, \overline{v^{\prime} u^{\prime}}, \overline{v^{\prime} \theta^{\prime}}, \overline{v^{\prime} \varepsilon^{\prime}}, \overline{w^{\prime} \theta^{\prime}}$, and $\overline{w^{\prime} \varepsilon^{\prime}}$. We have seen that $\overline{v^{\prime} \theta^{\prime}}$ is generally parametrized by the Austauch approach. Stone (1973) showed how sensitive the results can be for such a parametrization. Sometimes constant Austauch assumption can be very misleading. His results are reproduced in figure 8. At this point we shall digress a little and dwell on the important aspects of the large scale eddy flux parametrization.

In almost all such parametrizations, the baratropic baroclinic instability theory is invoked in general. Baroclinic linear instability theory gives us the fastest growing mode, its rate of growth and its structure in the initial stages. Barotropic instability theory also does the same for barotropic case. In general, it is assumed that baroclinic instability analysis gives the unstable mode and this mode barotropically transfers momentum. It is also assumed, in general, that this unstable mode alone characterises all the transient eddies. Though the instability theory gives the gravest mode, growth rate, and structure, it leaves the amplitude arbitrary. But the amplitude is essential to compute the transient fluxes. Therefore, to fix the amplitudes generally, some closure assumptions are introduced as in turbulence theory. A number of workers, Arakawa (1961), Saltzman and Vernekar (1971), Smagorinsky (1964), Sela and Wiin-Nielsen (1971), Kurihara (1973), Egger (1975), Greene (1970), Sasamori (1978) and Branscome (1983) to name some, arrived at various parametrization formulae. A few of these parametrizations are given in table 6.

One can see that finally, especially for energy fluxes, Austauch-type parametrizations are arrived at. Such formulae may not hold good locally because the scale of the eddy must be small when compared with the scale of the mean field, for

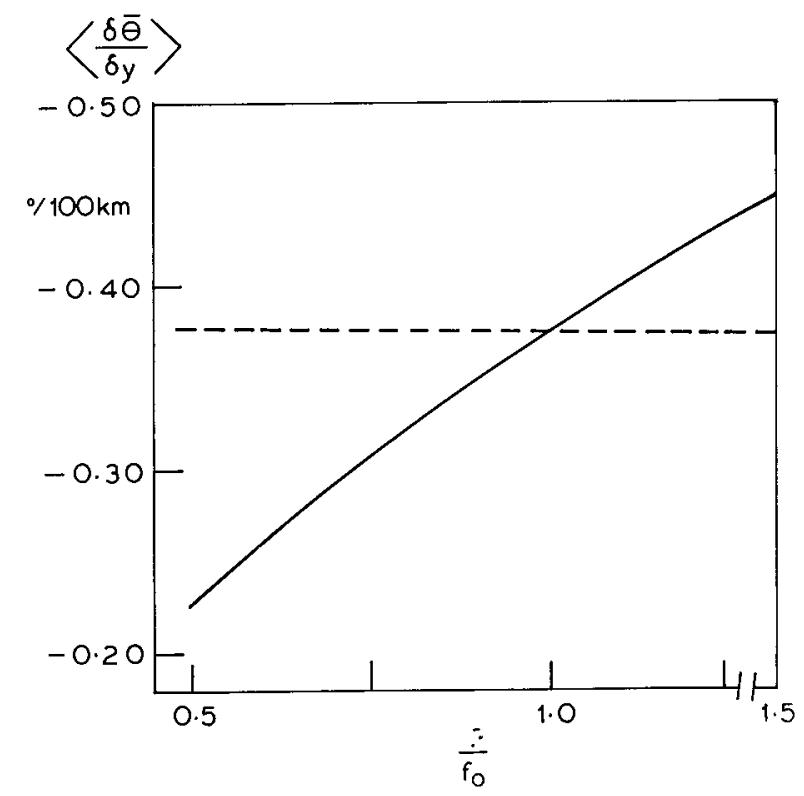

Figure 8. Mean horizontal temperature gradient vs. normalized values of the rotation rate. Solid: Calculations with non-constant eddy coefficients; dashed; that with constant eddy coefficients (from Stone, 1973). 
Table 6. Few large scale eddy flux parametrization schemes

Author(s) and Equations

Arakawa (1961)

$$
\begin{gathered}
\frac{\partial}{\partial t}{\overline{u^{\prime 2}}}^{t x}+2\left(1-\frac{2 D^{2}}{L^{2}}\right) \frac{\partial \bar{u}^{t x}}{\partial y}{\overline{u^{\prime} v^{\prime}}}^{t x}=0 \\
\frac{\partial}{\partial t}{\overline{v^{\prime 2}}}^{t x}+\frac{4 D^{2}}{L^{2}} \frac{\partial \tilde{u}^{t x}}{\partial y} \overline{u^{\prime} v^{\prime}} t x=0 \\
\frac{\partial}{\partial t} \overline{u^{\prime} v^{\prime}} t x+D^{2} \frac{\partial^{2} \bar{u}^{t x}}{\partial y^{2}} \frac{\partial}{\partial y} \overline{v^{\prime 2}} t x \\
\left.+\frac{\partial \bar{u}^{t x}\left(\overline{v^{\prime 2}}\right.}{\partial y}+\frac{2 x D^{2}\left({\overline{u^{\prime 2}}}^{t x}-\overline{v^{\prime 2}}\right)}{L^{2}}\right)=0
\end{gathered}
$$

Barotropic vorticity equation which conserves the absolute vorticity is the basic equation. $L$ and $D$, scale lengths of the disturbance in the $x$ direction and that of $v^{\prime} \partial \bar{u} / \partial y$ respectively are given by other considerations explicitly.

$$
\begin{aligned}
& \text { Saltzman and Vernekar (1971) } \\
& \overline{v^{\prime 2}}=-\Lambda \hat{\eta} S_{0}^{-1} \frac{1}{a} \frac{\partial \bar{\theta}^{x}}{\partial \phi} \\
& {\overline{v^{\prime} \theta^{\prime}}}^{x}=-K \frac{1}{a} \frac{\partial \bar{\theta}^{x}}{\partial \phi} \\
& {\overline{\omega^{\prime} \theta^{\prime}}}^{x}=\Pi K \frac{1}{a} \frac{\partial \bar{\theta}^{x}}{\partial \phi}\left(\frac{\partial \vec{\theta}^{x}}{\partial p}\right)^{-1} \\
& \overline{u^{\prime} v^{\prime}} x=T{\overline{v^{\prime 2}}}^{x} \cos \phi \frac{\mathrm{d} \mu}{\mathrm{d} \phi} \\
& \mu=\frac{\bar{u}^{x}}{a \cos \phi}-\frac{a \cos \phi}{n_{*}^{2}}\left(\beta-\frac{1}{a^{2}} \frac{\partial}{\partial \phi} \frac{1}{\cos \phi} \frac{\partial}{\partial \phi} \bar{u}^{x} \cos \phi\right) \\
& K \propto \frac{\partial \bar{\theta}^{t x}}{\partial p}\left(\frac{1}{a} \frac{\partial \bar{\theta}^{\ell x}}{\partial \phi}\right)^{-2}
\end{aligned}
$$

Barotropic and baroclinic instability theories are used. To determine $K$ and $T$ areally averaged conservation of energy principle and zonally averaged conservation of angular momentum principles are utilised. The gravest mode is determined as baroclinically the most unstable one Here $\theta$ is the potential temperature, $\Pi$ a constant $(0.75)$, and $T$ a characteristic time for wave tilting. $\Lambda$ is the constant of proportionality between the gravest mode growth rate and the kinetic energy of the unstable eddy. $K$ is determined by requiring that the upward heat flux below the level must balance the heat loss above. So is a static stability parameter proportional to $\left(\partial \theta_{0} / \partial p\right)^{1 / 2}$.

Greene (1970)

$$
\begin{aligned}
& {\overline{v^{\prime} S^{\prime}}}^{x}=-K_{v y} \frac{\partial \bar{S}^{x}}{\partial y}-K_{v z} \frac{\partial \bar{S}^{x}}{\partial z} \\
& {\overline{v^{\prime} S^{\prime}}}^{x}=-K_{z y} \frac{\partial \bar{S}^{x}}{\partial y}-K_{w z} \frac{\partial \bar{S}^{x}}{\partial z} \\
& \frac{\partial}{\partial y}\left(\rho_{0}{\overline{u^{\prime} v^{\prime}}}^{x}\right)=\rho_{0} K_{v y} \frac{\partial}{\partial y}(f+\xi) \\
& -\frac{f \rho_{0}}{B} \frac{\partial \phi}{\partial y} \frac{\partial}{\partial z} K_{v y}-f \frac{\partial}{\partial z} \rho_{0} K_{v z} \\
& \frac{\partial}{\partial y}\left(\rho_{0}{\overline{u^{\prime} v^{\prime}}}^{x}\right)=f \frac{\partial}{\partial z} \rho_{0} \overline{v \phi}^{x}-\frac{\rho_{0}}{B} \rho_{0}{\overline{v^{\prime} Q^{\prime}}}^{x}
\end{aligned}
$$

Here $Q=$ potential vorticity. For momentum transfer the barotropic theory of conservation of geostrophic absolute vorticity was used. An equation connecting ${\overline{Q^{\prime} v^{\prime}}}^{x}$ of ${\overline{u^{\prime} v^{\prime}}}^{x}$ which was utilized by Arakawa (1961) and Charney \& Stern (1962) was utilized. $K_{v z}, K_{w y}$ and $K_{w z}$ are proportional to $K_{v y}$. Kinematic consideration that $\frac{v y}{v^{\prime} Q^{\prime}}{ }^{x}$ is maximum at quarter period of the baroclinically unstable wave, was utilised. Here $C_{p} \phi$ is entropy and 
Table 6 (Contd.)

Author(s) and Equations

$Q^{\prime}=f \frac{\partial \phi^{\prime}}{\partial z}+B \xi+\frac{f}{\rho_{0}} \frac{\partial \rho_{0}}{\partial z}$

$K_{v y}=-1 \cdot 5 \alpha(g / B)^{1 / 2}(\Delta \phi)^{2} /(\partial \phi / \partial y)$
Comments

$B$ a static stability parameter. $S$ is any conservative parameter, $\phi=\log \theta$, and $\theta$ is potential temperature.

Branscome, L. E.

${\overline{v^{\prime} T^{\prime}}}^{\prime x}=0 \cdot 6 \frac{N d^{2}}{\alpha g}\left(\frac{\partial \bar{u}^{\prime x}}{\partial z}\right)^{2} e^{-(z / d)}$

Baroclinic instability theory used with

$d=\frac{h H}{h+H}$

$h=\frac{f^{2} \frac{\partial \bar{u}^{t x}}{\partial z}}{\beta N^{2}}$

${\overline{w^{\prime} T^{\prime}}}^{t x}=\frac{f}{N^{2}} \frac{\partial \bar{u}^{t x}}{\partial z}\left(\frac{z}{d}-\frac{z^{2}}{4 d^{2}}\right){\overline{v^{\prime} T^{\prime}}}^{t x}$

(variation of coriolis parameter $\beta$ ) effect explicitly included. $d$ is the characteristic depth of the unstable eddy, $N$ the Brunt Vaisala frequency, $\alpha$ the coefficient of thermal expansion and $H$ the density scale height. This parametrization holds good for both deep $(h \rightarrow H)$ and shallow $(h \rightarrow 0)$ waves. Note that $\overline{v^{\prime} T^{\prime}}$ depends on stability parameter $N$, just as in Saltzman-Vernekar equations it depends on $\left(\partial \theta_{0} / \partial p\right)$ through $K$.

\section{Sasamori}

${\overline{v^{\prime} T^{\prime}}}^{x}=-K_{y} \frac{\partial \bar{T}^{x}}{\partial y}$

$K_{y}=-\frac{0.067 \alpha_{3} R}{f . l_{e}} \frac{\partial \bar{T}}{\partial y}$

$l_{e}=\frac{f}{\left[R \sigma / 2\left(R / C_{p}\right)+1\right]^{1 / 2}}$

Geostrophic turbulence theory of Charney (1971) which shows that the large scale turbulence below a critical scale of Rossby radius of deformation, if properly scaled in the vertical, is analogous to the two-dimensional turbulence, was utilised. $l_{e}$ is the baroclinically unstable excitation wavelength.

Lorenz

${\overline{v^{\prime} T^{\prime}}}^{x}=-K \frac{\partial \bar{T}^{x}}{\partial y}+r$

After studying real data extensively Lorenz (1979) suggested this type of formulae where $r$ is a random parameter.

Austauch approach to be correct locally. It was observationally found by many workers that such parametrizations are not good enough over certain regions (Shaw 1966; Srivatsangam 1978; and Lorenz 1979). Nevertheless these parametrizations are based on physical theoretical knowledge and can be considered as definite advances in a problem which is no less difficult than the problem of turbulence. 
It must be noted that we have given only a few typical parametrization schemes. Other schemes which are equally important are not given here for the sake of brevity. With this brief digression we now return to the zonally averaged two-dimensional models. A typical model is that of Saltzman and Vernekar (1971).

Zonally-averaged two-dimensional steady state model of Saltzman and Vernekar: Saltzman and Vernekar (1971) start with the primitive equations in the $p$ system given symbolically as

$$
\begin{aligned}
& \mathrm{d} \Phi_{i} / \mathrm{d} t=X_{i} ; i=1,4, \\
& \Phi_{1}=u, \Phi_{2}=\nu, \Phi_{3}=\theta, \Phi_{4}=\varepsilon, \\
& \partial \phi / \partial p=-\frac{R \Phi_{3}}{P}\left(\frac{P}{P_{\mathrm{S}}}\right)^{\kappa} ;\left(\text { Here } \phi \text { is geopotential and } \kappa=R / C_{p}\right) \\
& \frac{1}{a \cos \phi} \frac{\partial \Phi_{1}}{\partial \phi}+\frac{1}{a \cos \phi} \frac{\partial}{\partial \phi} \Phi_{2} \cos \phi+\frac{\partial \omega}{\partial p}=0 .
\end{aligned}
$$

From this set they get another set describing the zonally symmetric time averaged state. Steady state equations of such a set were the starting point.

The parametrization of the radiative and small scale turbulent fluxes by Saltzman and Vernekar can be compared to that of Sellers. However, their parametrizations of large scale fluxes are different and are given in table 6 . They solved the equations just as Sellers did. Though the vertical structure is assumed, this model is considered as the two-dimensional model; because the atmosphere and ocean are considered separately and the model can be viewed as a two-layer system model. They finally arrive at an equation governing the vertically integrated temperature of the atmosphere. This is a disguised diffusion equation (having advection clubbed with forcing) given by

$$
\frac{\mathrm{d}}{\cos \phi \mathrm{d} \phi} K_{\times} \cos \phi \frac{\partial \bar{\theta}^{x z}}{\partial \phi}-\lambda(\phi) \bar{\theta}^{x z}=-F_{0}(\phi)
$$

Here $K_{x}$ is the nonconstant Austauch coefficient, $\lambda(\phi)$ involves atmospheric radiative parameters and $F_{0}(\phi)$ is the forcing due to radiative fluxes and fluxes due to zonally averaged and zonally asymmetric motions. In (29), $K_{\times}$is not a constant but a function of surface winds and temperature. For surface parameters they arrive at another system that gives $u_{0 S}, v_{0 S}$ and $\theta_{0 S}$. To arrive at the surface parameters the authors utilised the absolute angular momentum balance due to frictional forces and large scale momentum flux convergences.

Saltzman and Vernekar solved such a system iteratively within about 100 iterations. The amount of success of such a method can be seen from figure 9 . The authors applied this model to predict the temperature of the earth atmospheric system due to realistic changes in solar radiation to test the theory of Milankowich (1930) and found only little variation. Including cloud albedo feedback later they found the system similar but more stable than the Budyko-Sellers system.

With a diffuse cloudiness feedback mechanism, Temkin and Snell (1976) worked with a similar zonally symmetric model utilizing Austauch approach for eddy flux terms. The response of this model for the standard test runs, doubling of $\mathrm{CO}_{2}$ and reduction of solar constant by $1 \%$, was found to be more than that of global model but lesser than that of other workers. We give a part of their results in table 7 . Even in this model we see from table 7 that the diffuse cloudiness feedback makes the 


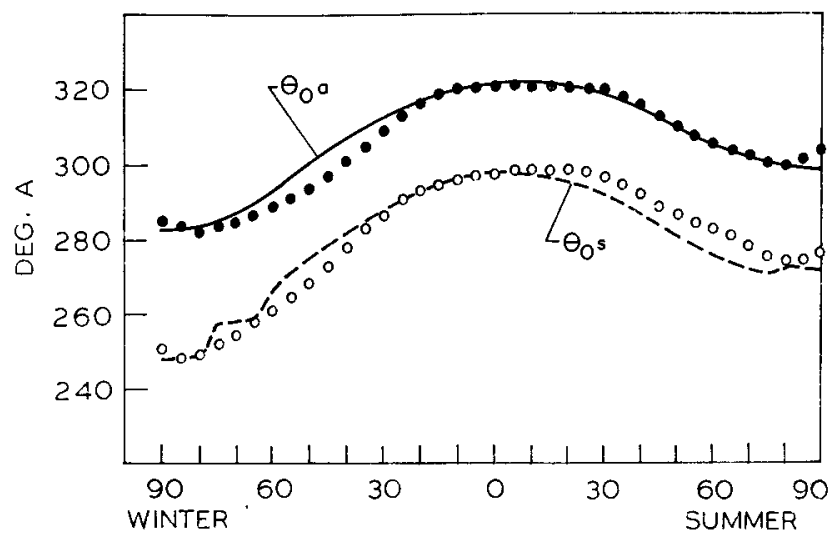

Figure 9. Mean latitudinal distribution of the surface potential temperature $\theta_{\mathrm{os}}$ (lower curve) and vertically averaged potential temperature $\theta_{\text {oa }}$ (upper curve). Black and white circles represent the observed values (from Saltzman and Vernekar, 1971).

Table 7. Global annual sea level temperature change $(\mathrm{K})$ for given environmental variation.

\begin{tabular}{lrrrrcc}
\hline & Zonal & Global & Budyko \\
Variation & model & model & Sellers \\
$(1973)$ & $\begin{array}{c}\text { Manabe and } \\
\text { Weatherald } \\
(1975)\end{array}$ & $\begin{array}{c}\text { Rasool } \\
\text { Schneider } \\
(1971)\end{array}$ \\
\hline $2 \mathrm{CO}_{2}$ & 1.7 & 0.7 & $>4$ & 1.32 & 2.36 & 0.8 \\
$0.99 \mathrm{~S}$ & -1.0 & -0.7 & -5 & -5.00 & -1.30 & \\
\hline
\end{tabular}

system less sensitive. These results clearly bring about the importance of cloudiness feedback even over very long climatic scales.

In the above modèls of Sellers, Saltzman and Vernekar and Temkin and Snell, the atmosphere is explicitly considered. But the integrations could be performed with almost a time step of one month. The atmospheric equations contain gravity waves and one may wonder how such integrations were possible. In these models not only geostrophy is utilized consistently so that the gravity waves do not exist but also the diffusion number corresponding to the Austauch coefficient, $K_{*} \simeq 3 \times 10^{6} \mathrm{~m}^{2}$ $\mathrm{sec}^{-1}$ and the grid spacing of about $10^{6} \mathrm{~m}$, allows $\Delta t$ of that order. Thus these models could be utilized for palaeoclimatic integrations for thousands of years.

Adem (1963, 1964, 1965), developed similar thermodynamic models for the forecast of climate on monthly basis, and these models are being used for palaeoclimatic integrations. The basic idea of parametrizing entire dynamics was mooted by Adem as early as sixties. The diffuisive model of Adem (1981) could give results similar to those of Gates (1976).

Our discussion of the energy balance models will not be complete without mentioning a highly interesting idea due to Paltridge (1975). Working with the energy balance models Paltridge found that the earth-atmospheric climate system reaches a steady state such that the global net rate of production of entropy (which is the sum over all latitude zones of the ratio of the net radiant energy input to the 
effective emission temperature of the zone) is minimized. The model based on this principle shows greater stability than'Budyko-Sellers original models. Very limited work is reported on these variational type of models. (North et al 1979).

We have seen that most of the palaeoclimatic models are highly parametrized. If the parametrizations are exactly true, it will be ideal. However, if they are not yet perfected it is important to check the predictions of these simple toy models with full three-dimensional general calculation models in which structural parameters are minimum. However, the CFL criterion prohibits palaeoclimatic calculation with these models until a super computer is developed. But these models can be utilized to test the effect of the changed boundary conditions and changed composition of the atmosphere, though they cannot be utilized to simulate very long time scale phenomena such as the onset of a "White Earth" because of the huge cost involved. We shall now deal again very briefly with a few results of few experiments with GCMs.

\section{Three-dimensional GCM type models}

The GCMs currently operating at various centres such as GLAS, (essentially the UCLA Arakawa-Mintz model is further developed here by Shukla, Halem, Kalney and others), GFDL, (the model developed by Smagorinsky, Manabe, Miyakoda, Bryan, Kurihara and others is used here), NCAR (a model developed by Kurihara and Washington is in operation here), etc. are of two kinds basically, depending on how the horizontal advection and diffusion terms are treated. Besides these complex models, there are many other models such as that of Webster and Lau (1977) which are ocean atmosphere models that are less complex. The two kinds of GCMs are (1) discrete grid type and (2) spectral type. In these models, there are basically five important parts: (i) the skeleton advection diffusion equation part, (ii) radiation part, (iii) boundary layer part, (iv) convective parametrization part and (v) ocean part and hydrology part. Even in these models, especially in parts (ii), (iii) and (iv) there is a large amount of parametrization involved. All these models are almost equally successful in explaining the present-day climate. As a sample of the capability of these models, in the detail that they can provide, we give in figure 10 , the zonal structure of the atmosphere given by some of these models in some experiments.

The most important aspects of GCMs when compared with any other climate model are the following, regarding each of the above-said five parts in that order:

(i) The dynamics is considered explicitly by the well-known fluid mechanical equations based on conservation of momentum, mass and energy principles. The scales explicitly considered are truncated only by the hydrostatic limit. Even the finite difference approximations to these equations are constrained to conserve mass, momentum (in some models), enstrophy and energy, globally. Because of this fundamental approach the advection of energy in these models is the result of natural evolution of hydrostatic meteorological systems and so is definitely more physically satisfactory. However, because of this approach these models are most complex and computer time-consuming too. For instance, at present the integration of a GCM for over few hundred years is considered unaffordable even 


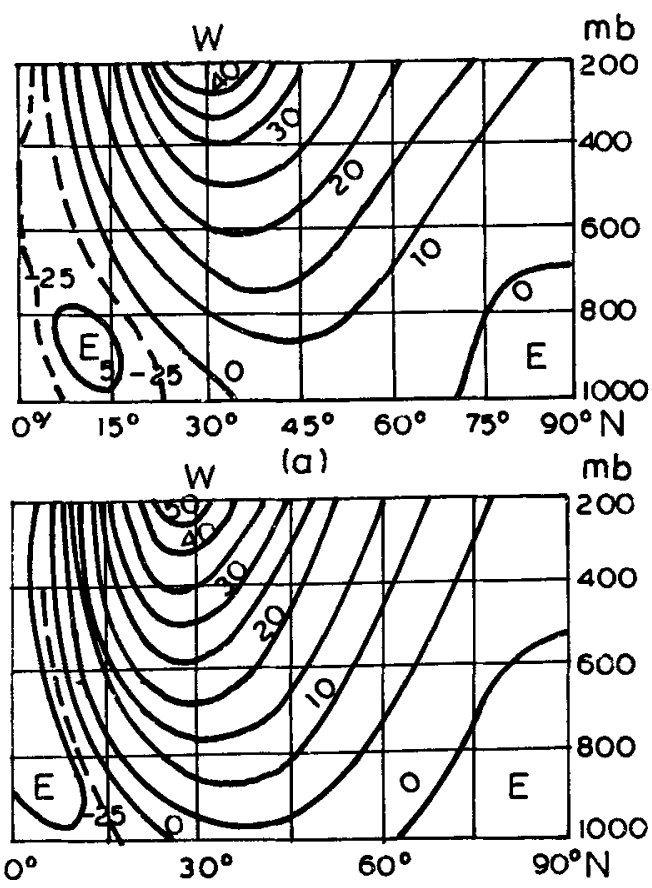

(b)

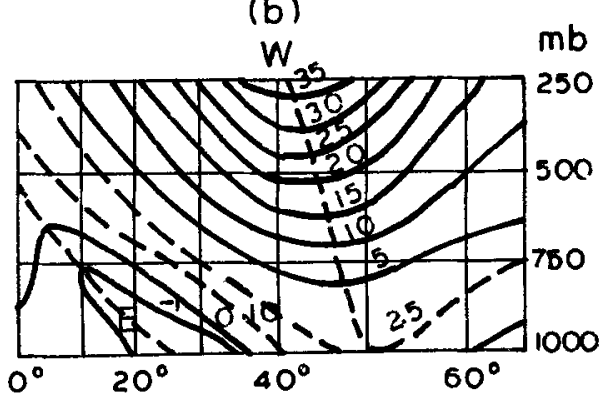

(c)

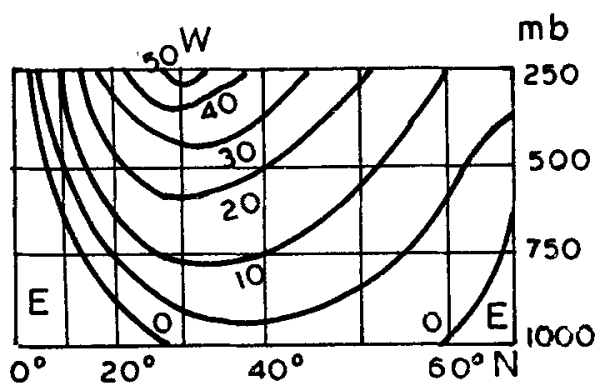

(d)

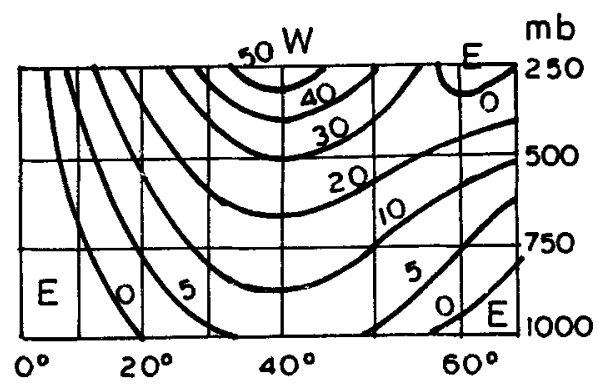

(e)

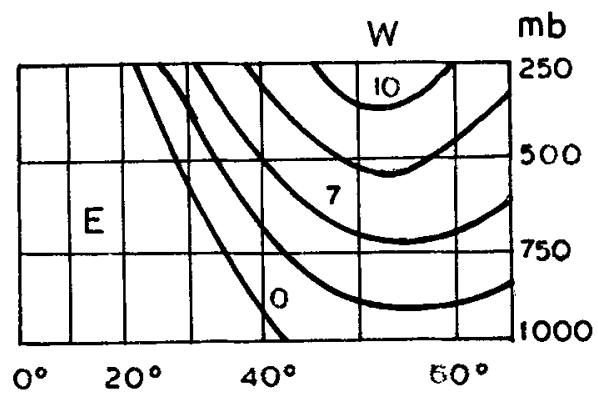

(f)

Figure 10. Distribution of the mean zonal wind in the Northern Hemisphere a) the observed mean for winter, b) mean for 30 days - Mintz, c) mean for the period from the 17 th to the 39 th day - Smagorinsky, d) mean for 26 days - Charney, e) mean for the period from the 8 th to the 30 th day - Phillips, f) mean for winter - Adem (from Gavrilin 1965).

for the richest country in the world. The time step used is of the order of about $10 \mathrm{~min}$ to $1 \mathrm{hr}$ for $500 \mathrm{~km}$ resolution and about 2000 points have to be considered over the globe. If the number of variables is 6 and the vertical levels about 10 in general, the computer time required can easily be imagined.

(ii) In radiation computations, the radiative transfer of $\mathrm{CO}_{2}, \mathrm{O}_{3}$ and $\mathrm{H}_{2} \mathrm{O}$ is considered in great detail. The vertical resolution as already indicated is about 10 levels. $\mathrm{CO}_{2}$ and $\mathrm{O}_{3}$ are generally fixed in time and space. Because $\mathrm{H}_{2} \mathrm{O}$ varies in time in these models which consider the conservation of water vapour equation also, the surface temperature humidity feedback is automatically taken care of. 
Because of the high cost of radiative computations, these are performed only once in every five hours and the radiative heating is kept constant in between. In many models at present, clouds too change with time. The clouds considered are in general three types; low, medium and high and are black in the infra-red. Short wave cloud albedo is a function of the cloud type and is taken from observations. (iii) The planetary boundary layer processes in GCMs are parametrized in equally detailed manner. To take care of the transfer processes during stable, neutral and unstable conditions semi-empirical theories are utilized.

In the surface layer at the interface the fluxes are determined, in general, by the aerodynamic type formulae in many of the models. For instance, the evaporation depends on the specific humidity difference between the lower boundary and the lowest model layer. The heat flux depends on the temperature difference. The momentum flux depends on the velocity difference. The roughness length appears in all the formulae.

(iv) The precipitation is generally considered to be of two types: (a) large scale precipitation-this occurs when the grid point of the model is supersaturated. The precipitation is proportional to the difference between the specific humidity at the grid point and the saturation specific humidity. In many cases, saturation is deemed to occur when relative humidity is about $80 \%$. The rainfall depends on such thresholds. These thresholds are generally tuned to observations. (b) Convective precipitation-This occurs mainly in tropics due to what is known as the convective instability of the second kind (CISK). Because of large humidity in tropical boundary layer, the atmosphere if lifted to a level (lifting condensation level), becomes unstable due to release of latent heat. This lifting can occur due to Ekman frictional convergence, which in turn can occur due to cyclonic system above the boundary layer. When such lifting occurs, large amount of moisture from the boundary layer goes into the free atmosphere because of instability, precipitates there and heats the atmosphere. This process can also transfer momentum. It is found that especially for tropical motions this phenomenon is extremely important. Hence in GCMs, this convection is parametrized in different ways. ArakawaSchubert parametrization is the most complex of all such parametrizations. It computes a spectrum of clouds. Kuo's parametrization is much simpler. Finally, the convective adjustment method of GFDL model is the simplest. It adjusts the whole column such that there is no more instability and in that process, precipitates some water substance. The model climate depends very much on tropical climate. If the convection is strong, the Hadley circulation will be strong and this in turn effects the whole global circulation. One can see that this convective parametrization not only redistributes heat, moisture and momentum in the vertical but also effects the surface heat, moisture and momentum fluxes via the precipitation induced dynamics.

(v) In many of the models ocean is treated as a swamp without motion. Some ocean-coupled models have the surface layer of the ocean driven by the wind stress. In ocean-coupled models, ocean model is integrated with larger time steps than the atmosphere. Atmospheric forcing is kept constant for each such large step. In general, the depth of the snow cover, and the amount of soil moisture over continents are computed by detailed balance requirements. Snow depth increases by snowfall and decreases due to ablation. The amount of ablation is computed by heat balance requirements when snow melt conditions are fulfilled. Difference 
between rain and snow is determined by temperature at about 400 meters. If this is below zero, snowfall occurs.

Thus we can easily conclude that the GCMs are the most sophisticated tools for climate study but are still very costly for the very long term phenomena.

A number of experiments on the sensitivity of the climate on a time scale of a month, to various external and internal anomalies are being conducted with these models. One of the most important factors of influence on this scale is the SST according to some observations e.g. Bjerknes (1966), Rowntree (1972, 1976), Namias (1964). A number of experiments were conducted with these models, to understand the effect of SST anomaly (SSTA) on the climate on this time scale. The main difficulty is the fact that the SST anomalies (SSTA) are of the order of $1^{\circ} \mathrm{C}$ and their effect in general is submerged in the noise of these GCMs. However when experiments were performed with stronger forcings (SSTA $>2^{\circ} \mathrm{C}$ to $3^{\circ} \mathrm{C}$ ), the models predict significant changes in the climate on this time scale e.g. Shukla (1975). To circumvent the noise problem linear models are being utilized e.g. Egger (1977), Webster (1972). The linear models have the problem of resonance for certain scales. However the overall impression that one gets after perusing the results of all these SSTA observations and experiments is that there cannot be any doubt about the effect of SSTA on climate on this time scale. These effects need not confine to the region of SSTA and in many cases they can propagate to higher latitudes by complicated teleconnecting dynamical processes such as induced propagating Rossby waves e.g. Hoskins and Karoly (1981).

A number of palaeoclimatic boundary conditions and composition sensitivity experiments are being conducted with some of these models e.g. Williams (1974), Williams et al (1974), Wetherald and Manabe (1975), Gates (1976). The boundary conditions for these experiments correspond to $18000 \mathrm{YBP}$, when a glacial age took place and for which there is enough evidence. These palaeociimatically reconstructed data indicate that the ocean was several degrees colder during such an ice age (CLIMAP). The great ice sheets covered the continents upto nearly $50^{\circ}$ latitude from the poles. The computations are generally made with such boundary conditions. We note that these types of computations do not give us any idea of how such boundary conditions resulted in the first place. Instead they tell us how the atmosphere was balanced for those boundary conditions.

A typical example of such a palaeoclimatic experiment with a simple GCM is that of Gates. Many interesting results were obtained when one looks at the details of fluxes, energetics and fields. Here we give only one result, that of the mean zonal temperature obtained for such boundary conditions, in figure 11 . One can see that everywhere the ice age temperature is colder. This only reflects the boundary temperature conditions. Such results indicate that one can treat the atmosphere as a quick response system to the lower boundary (Saltzman). In the polar regions, there is an indication of slight increase in baroclinicity. All these results depend on the tropical ocean temperature which controls baroclinicity.

Manabe and Wetherald $(1975,1980)$ conducted $\mathrm{CO}_{2}$ and cloud sensitivity experiments with the GCM of the GFDL, which includes the detailed hydrological cycle. Two surprising results emerge from these studies: (1) The poleward transport of moist static energy is independent of the solar radiation variations between $+2 \%$ to $-4 \%$. (2) The variable clouds have insignificant effect when compared with fixed clouds on the total net radiation into the system. Both the 


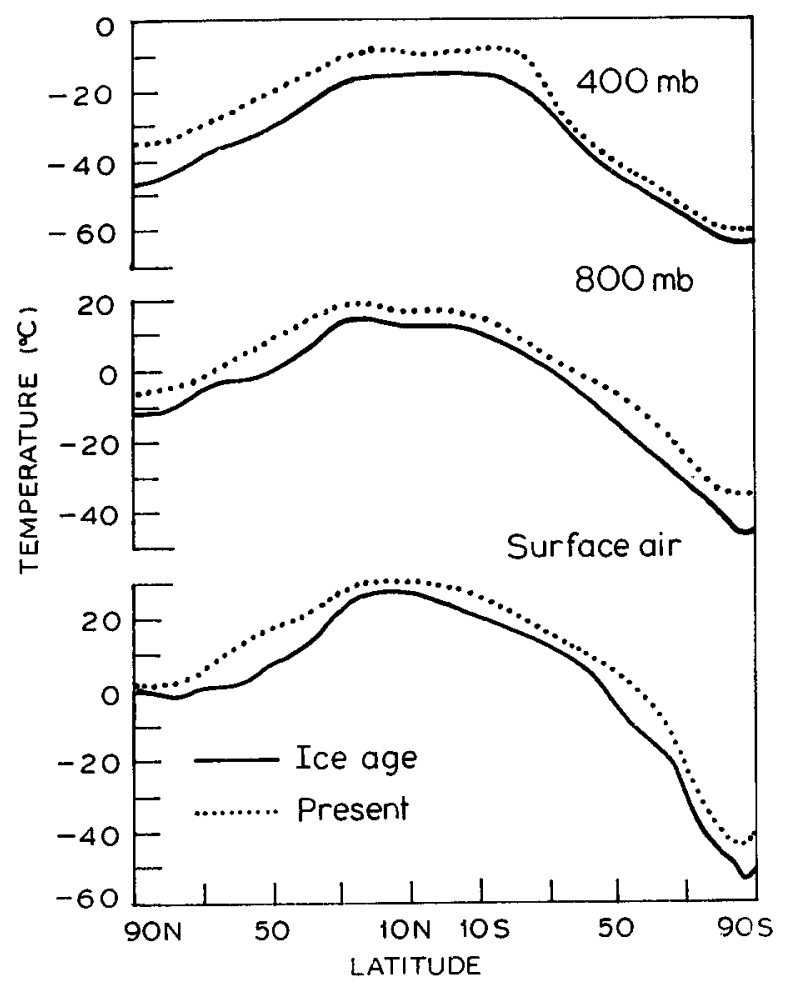

Figure 11. The zonally averaged $400 \mathrm{mb}, 800 \mathrm{mb}$ and surface air temperature simulated for the ice age and present July (from Gates, 1976).

above results are the result of compensating effects. However, these results have to be checked with other models because such results critically depend on parametrizations. For instance, Shukla and Sud (1981) report significant effect of variable cloudiness on seasonal climate.

Held and Suarez (1978) are experimenting with simple highly truncated GCMs for climatic sensitivity. Their results show that the inclusion of only one unstable wave results in reasonably realistic zonal mean fields e.g. figure 12 . Inclusion of one or two more waves resulted in marginal changes such as the destruction of polar jet. These results along with other interesting results obtained by the authors indicate that the large scale eddy flux parametrization that depends on only one gravest mode that is unstable baroclinically can be realistic. No wonder these parametrizations are remarkably successful.

\section{Conclusions}

The calculations by a widely different spectrum of models indicate that the earth atmospheric system is quite stable for small perturbations in all parameters including solar radiation. At least a $2 \%$ decrease in solar radiation is necessary to produce a white earth. The decrease can be anywhere between $2 \%$ and $20 \%$ e.g. Warren and Schneider (1979). The hierarchy of importance in the parameters at 


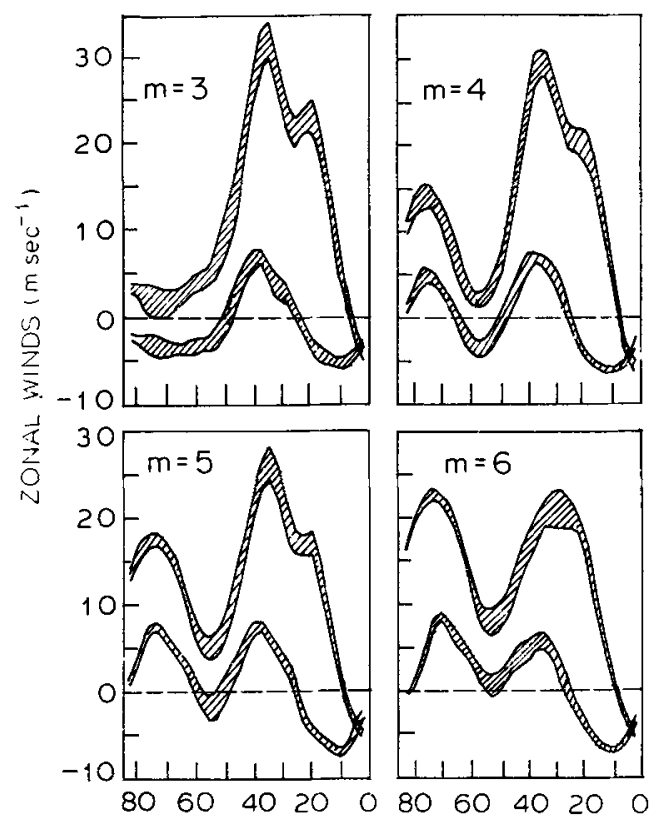

Figure 12. Upper and lower layer zonal winds produced by moist 1-wave models with $\mathrm{m}=3,4,5$ and 6 . Estimated standard deviations of 200-day averages are shown in each case (from Held and Suarez, 1978).

present seems to be (i) solar radiation (ii) $\mathrm{CO}_{2}$ (iii) Clouds and (iv) Aerosols. However the hierarchy is not yet very clear.

Can such changes occur in the sun? If so can we predict them well? These are questions that meteorologists may have to ponder in future. Can such effective decrease in solar radiation reaching the ground occur due to spreading of dust either by volcanic eruptions or by nuclear wars is another question. Next in importance is the cloud. The future climate models must be able to parametrize the cloud feedback mechanism realistically. To arrive at correct results the physics of clouds must be continuously understood for this reason. The aerosols take the last place in importance. However, over a long time scale these may be of great importance, especially in upper troposphere and stratosphere. In introducing the effects of aerosols such as sulphates, dust, salts and organic compounds, into climatic models, their natural cycles must be studied observationally, understood and then incorporated in future. Their scattering properties, their effects on precipitation and cloud albedo must be studied observationally for a long time to come to understand their direct and indirect importance for climate changes.

There seem to be natural oscillations in the earth atmospheric system according to some models (Saltzman). According to one simple model with only three components, deep continental ice sheet mass, marine ice mass and the mean world ocean temperature, Saltzman et al (1984) could get $100 \mathrm{ky}$ cycles, without any external forcing having such a long period. The nonlinear system behaviour is involved here. The understanding of such oscillation is as important as it is to understand a steady state. With the astronomical forcings, the structure, the variance spectrum and the phase of the quaternary glaciations were obtained. 
Imbrie (1980) also obtained with a highly simplified model some support for the astronomical theory of climate on these scales. However, it is too early to come to firm conclusions. By reconstructing the geologic history of the earth atmospheric system we might be able to collect more observational evidence regarding such oscillations. Therefore this must be our endless goal. With the help of these models can we start at one epoch and predict correctly the next observed epoch in such a set of observation will be the next question.

Thus we see that two broad hypotheses of climate change exist today. The first one, the Milankowichian hypothesis says that the perturbation in the earth's orbital parameters due to astronomical causes, changes the zonal distribution of the incoming solar radiation though not its total global magnitude. Warm summers and mild winters over the critical ice latitudes result in deglaciation and vice versa. The periods of such perturbations are in the same range as the ice ages and so the hypothesis is plausible. The second one, the internal oscillation hypothesis, probes the idea that the system oscillates due to internal feedbacks. The time periods of such oscillations are also in the same range as the ice ages. The tussle goes on and the final judgement is not in sight yet.

Thus the overall feeling we get at this juncture is that we have a long way to go before we understand correctly significant climatic changes, though we are not in the immediate danger of a deep freeze unless some large catastrophic change, on the earth or in the sun, occurs.

\section{Appendix A}

$a, C \quad$ : empirical constants derived and used by Sellers

$-B \quad$ : suffix indicating lower boundary of the ocean layer

$C_{m} \quad:$ mean specific heat

$C_{m 1}, C_{m 2}$ : specific heats of the atmosphere and oceans resp.

$\frac{\mathrm{d}}{\mathrm{d} t} \quad$ : total derivative

$a_{\nu} \quad:$ albedo of single scattering radiation

$\varepsilon \quad$ : water vapour mixing ratio

$F_{\mathrm{HH}} \quad$ : horizontal heat flux due to subgrid scale turbulent conduction

$F_{\mathrm{HV}} \quad$ : vertical heat flux due to small scale turbulent conduction

$F_{\mathrm{HH} x}, \quad: x$ and $y$ components of horizontal heat flux

$F_{\text {HHy }}$

$F_{\text {Hvo }}^{i}:$ component of the vertical heat flux at the interface

$\bar{F}_{\mathrm{HV} i}^{t x} \quad$ : component of the vertical heat flux, average over $t \& x$

$\gamma \quad$ : scattering coefficient

$\Phi \quad$ : geopotential

$\phi \quad$ : latitude

$K_{\nu} \quad$ : absorption coefficient

$L_{\nu} \quad$ : radiation intensity 


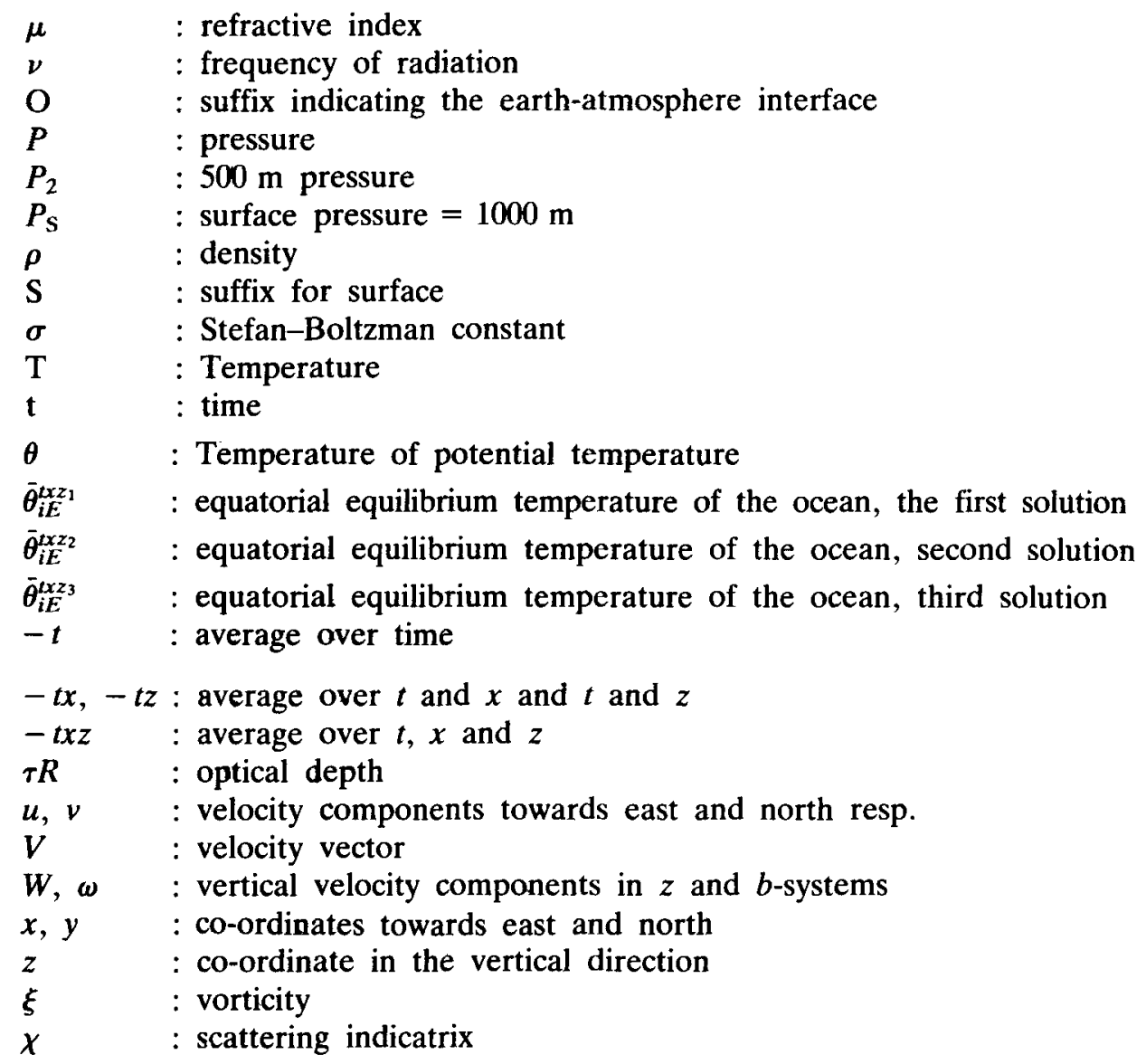

\section{Appendix B}

Each climate model is like a system in which advective heat transfer is a subsystem. If two or more than two types of parametrizations for one subsystem result in the same major output of the total system (such as white earth for about $1.6 \%$ decrease in solar radiation) we do not really know whether we should feel happy or disturbed. Similar results abound in meteorology. For instance, different parametrizations for surface fluxes give almost similar results for the mean state. Similarly differing cumulus parametrization do not give comparatively different results for the steady state. Such results seem to mean at least one of the following:

(i) The subsystem is unimportant. We may rule this out because we do not include it unless we know it is important.

(ii) The subsystem models, though they appear to be different, are not really different.

(iii) The subsystem models are really different, but the final output is independent of them, because of some deeper reason or because of tuning.

(iv) Though the final system output is the same, the actual history by which such a result is attained, may be quite different because the subsystem models are 
different. Not only that. Depending on the level of sophistication of the questions we ask not only the models may differ from each other in their answers, but also they may differ widely from the real nature, though they are all tuned to give the right answer for a particular question.

(v) The total system, in other words, the master model, is simply not suited to introduce the variations in the parametrizations of the subsystem. For instance, Cumulus parametrization is concerned with the vertical redistribution of heat and momentum. If the master model is not well resolved in the vertical, it cannot bring out the differences in the parametrization schemes, in the final model output.

Budyko parametrization corresponds to Rayleigh friction whereas Sellers parametrization is like the Navier-Stokes viscous effect. For smooth temperature profiles they are identical qualitatively if the diffusion coefficient is inversely proportional to the square of the wave number. For wave number one, the difference can only be in the actual values taken. For all wave numbers the Budyko time constant

$$
\left(\beta / C_{p} \int_{0}^{H} \rho \mathrm{d} z\right)
$$

is approximately 3 days. This corresponds to a scale of $500 \mathrm{~km}$ for Sellers diffusion $\left(K_{H}=10^{6} \mathrm{~m}^{2} \mathrm{sec}^{-1}\right)$. In the symmetric problem, North showed that wave number one $P_{2}(x)$ is the dominant one for the temperature most probably because of the net radiational forcing. On this scale there will be a large difference between, the time constants of Budyko and Sellers, the later one being much larger. Therefore, the transport mechanism is much weaker in the case of Sellers. We expect thus, much faster growth of ice and the onset of white earth in the case of Sellers model. In the case of Saltzman's model the temperature is a smooth function of latitude. This only means that much faster heat transport and so Saltzman's model is nearer to Budyko model. However, the sensible heat flux depends on baroclinicity in the Saltzman's model. In effect this might be looked at as a heat transport mechanism in which $K_{H}$ depends on temperature gradient very strongly. To the best of author's knowledge, the above aspects were seldom clarified in the literature.

This is only an attempt to compare certain aspects of these models. Evidently, it is a difficult problem to be further examined. From this point of view it appears to be more advantageous to build the subsystems within the framework of an already existing total system (instead of building ever new total systems) if it can be considered reasonably realistic.

\section{References}

Adem J 1963 Mon. Weather Rev. 91375

Adem J 1964 Mon. Weather Rev. 9291

Adem J 1965a J. Geophys. Res. 70376

Adem J 1965b Mon. Weather Rev. 9395

Adem J 1981 J. Geophys. Res. 8612015

Arakawa A 1961 J. Met. Soc. (Jpn) 3949

Bjerknes J 1966 Tellus 18820 
Bjerknes J 1969 Mon. Weather Rev. 97163

Branscome L E 1983 J. Atmos. Sci. 402508

Bryson A R and Dittberner G J 1976 J. Atmos. Sci. 332094

Budyko M I 1969 Tellus 21611

Bunker A F 1976 Mon. Weather Rev. 1041122

Cahalan R F and North G R $1979 \mathrm{~J}$. Atmos. Sci. 361178

Cess R D $1976 \mathrm{~J}$. Atmos. Sci. 331831

Charney J G 1971 J. Atmos. Sci. 281087

Charney J G and Stern M E 1962 J. Atmos. Sci. 19159

Chylek P and Coakley J A 1975 J. Atmos. Sci. 32675

Dwyer H A and Peterson T 1973 J. Appl. Meteorol. 1236

Egger J 1975 Tellus 27325

Egger J 1977 Mon. Weather Rev. 34603

Emiliani C 1955 J. Geol. 63

Faegre A 1972 J. Appl. Meteorol. 114

Fredriksen J S 1976 J. Atmos. Sci. 332267

Gates W L 1976 J. Atmos. Sci. 31844

Gavrilin B L 1965 Atmos. Ocean Phys. Series 11229

Ghil M 1976 J. Atmos. Sci. 333

Green J S A 1970 Q.J.R. Meteorol. Soc. 96157

Gribben J (ed.) 1978 Climate Change (Cambridge: University Press)

Hasselmann K 1976 Tellus 28473

Held I M and Suarez M J 1974 Tellus 26613

Held I M and Suarez M J 1978 J. Atmos. Sci. 35206

Hoskins and Karoly $1981 \mathrm{~J}$. Atmos. Sci. 381179

Imbrie J 1980 Math. Phys. Sci. NATO advanced study institute series (Series C) (ed.) A Berger p. 527

Kurihara Y 1973 J. Atmos. Sci. 3025

Lemke P 1977 Tellus 29385

Lindzen R S and Farrel B 1977 J. Atmos. Sci. 341487

Lorenz E N 1963 Trans. NY Acad. Sci. 25409

Lorenz E N 1979 J. Atmos. Sci. 361367

Manabe S and Moller F 1961 Mon. Weather Rev. 89503

Manabe S and Strickler R F 1964 J. Atmos. Sci. 24361

Manabe S and Wetherald R T 1967 J. Atmos. Sci. 24241

Manabe S and Wetherald R T 1980 J. Atmos. Sci. 3799

Milankovich M 1930 Koppen-Geiger Handb. Klimat (Berlin: I A) p. 176

Mokhov I I 1979 Atmos. Ocean Phys. Series 15258

Moritz R E 1979 J. Geophys. Res. 844916

Namias J 1964 Tellus 3394

Newell R E 1974 Q. Res. 4117

North G R 1975a J. Atmos. Sci. 321301

North G R 1975b J. Atmos. Sci. 322033

North G R and Coakeley J A 1979 J. Atmos. Sci. 361189

Paltridge G W 1975 Q. J. R. Met. Soc. 101475

Ramanathan V 1976 J. Atmos. Sci. 331330

Ramanathan V and Coakley J A 1978 Am. Geophys. Union 3465

Rasool S I and Schneider S H 1971 Science 173138

Reynolds R W 1978 Tellus 3097

Ruiz A D E and Lemke P 1982 Tellus 34313

Rowntree 1972 Q.J.R. Meteorol. Soc. 98290

Rowntree 1976 Q.J.R. Meteorol. Soc. 102607

Saltzman B 1978 Adv. Geophys. 20183

Saltzman B 1980 Arch. Met. Geophys. Biokl. 2941

Saltzman B and Moritz R E 1980 Tellus 3293

Saltzman B and Vernekar A D 1971 J. Geophys. Res. 761498

Saltzman B and Vernekar A D 1971 J. Geophys. Res. 764195

Saltzman B and Vernekar A D $1983 \mathrm{~J}$. Atmos. Sci. 401426

Saltzman B, Hansen A R and Kirk A H 1984 J. Atmos. Sci. 411 
Saltzman B et al 1981 J. Atmos. Sci. 38494

Sasamori T and Melgareio J W Tellus 30289

Schneider S H 1975 J. Atmos. Sci. 322060

Schneider S H and Dickinson R E 1974 Rev. Geophys. Space Phys. 2447

Schneider S H and Galchen T 1973 J. Geophys. Res. 786182

Schneider S H and Mass C 1975 WHO Technical note No. 21 p. 365

Sela J and Wiin-Nielsen A 1971 Mon. Weather Rev. 99460

Sellers W D 1969 J. Appl. Meteorol. 8392

Sellers W D 1973 J. Appl. Meteorol. 12241

Sellers W D 1976 Mon. Weather Rev. 104233

Shaw D B 1966 Q.J.R. Meteorol. Soc. 92

Shukla J 1975 J. Atmos. Sci. 32503

Shukla J and Sud Y 1981 J. Atmos. Sci. 382337

Smagorinsky J 1963 Mon. Weather Rev. 9199

Smagorinsky J, Manabe S and Holloway J L 1965 Mon. Weather Rev. 93727

Srivatsangam S $1978 \mathrm{~J}$. Atmos. Sci. 351212

Stone P H 1973 J. Atmos. Sci. 30521

Su C H and Hsieh D Y 1976 J. Atmos. Sci. 332273

Temkin R L and Snell F M 1976 J. Atmos. Sci. 331671

Urey H C 1947 J. Chem. Soc. Part I 562

Wang W C et al 1976 Science 194685

Warren S G and Schneider S H 1979 J. Atmos. Sci. 361377

Weare B C and Snell F M 1974 J. Atmos. Sci. 311725

Webster P J 1972 Mon. Weather Rev. 100518

Webster P J and Lau K A M W 1977 J. Atmos. Sci. 541063

Wetherald R T and Manabe S 1975 J. Atmos. Sci. 322044

Wetherald R T and Manabe S 1980 J. Atmos. Sci. 371485

Williams J 1974 (NCAR: Colorado)

Williams J, Barry R G and Washington W M $1974 \mathrm{~J}$. Appl. Meteorol. 13305 